\title{
Reaction-diffusion problems in cylinders with no invariance by translation. Part II: Monotone perturbations
}

by

\author{
François HAMEL
}

Université Paris VI, Laboratoire d'Analyse Numérique, 4, place Jussicu, 75252 Paris Cedex 05, France.

ABSTRACT. - The purpose of this work is the study of the existence and of a priori properties of solutions $(c, u)$ of the following reaction-diffusion equations in infinite cylinders $\Sigma=\mathbb{R} \times \omega$ with outward unit normal $\nu$ :

$$
\left\{\begin{array}{c}
\Delta u-\beta\left(x_{1}, y, c\right) \partial_{1} u+f\left(x_{1}, u\right)=0 \text { in } \Sigma \\
\partial_{\nu} u=0 \text { on } \partial \Sigma \\
u(-\infty, \cdot)=0, u(+\infty, \cdot)=1
\end{array}\right.
$$

The functions $-\beta$ and $f$ are given and are non decreasing in $x_{1}$. The results on the existence and on the necessary conditions are related to two "limit problems" as $x_{1} \rightarrow \pm \infty$.

Key words: Nonlinear PDE's, Monotonicity properties, Sub- and Supersolutions, Sliding method, Asymptotic behaviours.

RÉsumÉ. - Ce travail porte sur l'étude de l'existence et d'estimations a priori de solutions $(c, u)$ d'équations de réaction-diffusion dans des cylindres infinis $\Sigma=\mathbb{R} \times \omega$ de normale extérieure unitaire $\nu$ :

$$
\left\{\begin{array}{c}
\Delta u-\beta\left(x_{1}, y, c\right) \partial_{1} u+f\left(x_{1}, u\right)=0 \text { dans } \Sigma \\
\partial_{\nu} u=0 \text { sur } \partial \Sigma \\
u(-\infty, \cdot)=0, u(+\infty, \cdot)=1
\end{array}\right.
$$

A.M.S. Clasification: $35 \mathrm{~J} 60.35 \mathrm{Q} 35,35 \mathrm{P} 10,35 \mathrm{P} 30,76 \mathrm{Z} 05$. 
Les fonctions $-\beta$ et $f$ sont données et sont croissantes par rapport à $x_{1}$. Les résultats d'existence et les conditions nécessaires sont reliés à deux « problèmes limites » quand $x_{1} \rightarrow \pm \propto$.

\section{INTRODUCTION AND MAIN RESULTS}

The goal of this paper is to investigate some problems which are set in infinite cylinders $\Sigma=\left\{\left(x_{1}, y\right), x_{1} \in \mathbb{R}, y \in \omega\right\}$, where $\omega$ is a bounded domain in $\mathbb{R}^{N-1}$ with smooth boundary; one denotes by $\nu$ the outward unit normal to $\partial \omega$ or $\partial \Sigma$. We study semilinear elliptic equations

$$
\Delta u-\beta\left(x_{1}, y, c\right) \partial_{1} u+f\left(x_{1}, u\right)=0 \text { in } \Sigma
$$

with boundary conditions

$$
\left\{\begin{array}{c}
\partial_{\nu} u=0 \text { on } \partial \Sigma \\
u(-\infty, \cdot), u(+\infty, \cdot)=1
\end{array}\right.
$$

the unknowns are the real $c$ and the function $u$. Let us denote by $(P)$ the problem which includes the equation $(E)$ and the boundary conditions (B.C).

One denotes by $\partial_{1} u$ and $\partial_{\nu} u$ the derivatives of $u$ with respect to $x_{1}$ and $\nu$ respectively. The given function $\beta$ is continuous in all parameters, lipschitz-continuous and bounded in $\left(x_{1}, y\right)$ for any $c \in \mathbb{R}$. This function $\beta$ is systematically assumed to be non increasing in $x_{1}$ and to have limits $\beta_{ \pm}(y, c)$ as $x_{1} \rightarrow \pm \infty$, uniformly in $y$ and $c$. Moreover, one assumes that $\forall c<c^{\prime} \exists \epsilon>0$ such that $\forall y \in \bar{\omega}\left|\beta_{ \pm}(y, c)-\beta_{ \pm}\left(y, c^{\prime}\right)\right|>\epsilon$. Lastly, $\beta$ is increasing in $c$ and $\beta\left(x_{1}, y, c\right) \rightarrow \pm \infty$ as $c \rightarrow \pm \infty$ uniformly in $x_{1}$ and $y$. For instance, a natural situation is to consider functions $\beta$ of the form $\beta\left(x_{1}, y, c\right)=c+\alpha(y)+\gamma\left(x_{1}\right)$ where $\alpha$ is a given function on $\bar{\omega}$ and $\gamma$ is decreasing on $\mathbb{R}$.

The nonlinear given term $f\left(x_{1}, u\right)$ is lipschitz-continuous in $x_{1}$ and $u$, and is defined on $\mathbb{R} \times[0,1]$. Moreover, $f$ is non decreasing in $x_{1}$, has derivatives with respect to $x_{1}$ and $u$, and there exist derivable functions $f_{ \pm}$on $[0,1]$ such that $\lim _{x_{1} \rightarrow \pm \infty} f\left(x_{1}, u\right)=f_{ \pm}(u)$ and $f_{u}^{\prime}\left(x_{1}, u\right) \rightarrow f_{ \pm}^{\prime}(u)$ as $x_{1} \rightarrow \pm \infty$ (uniform limits). Besides, one assumes that $f\left(x_{1}, 0\right)=f\left(x_{1}, 1\right)=0$ for all $x_{1} \in \mathbb{R}$. 
Let us first state the motivation and some results for the case where the terms $\beta\left(x_{1}, y, c\right)$ and $f\left(x_{1}, u\right)$ actually do not depend on $x_{1}$. Problem $(P)$ reduces to an invariant by $x_{1}$-translation problem

$\left(P_{\text {inv }}\right)$

$$
\left\{\begin{array}{c}
\Delta u-\beta(y, c) \partial_{1} u+f(u)=0 \text { in } \Sigma \\
\partial_{\nu} u=0 \text { on } \partial \Sigma \\
u(-\infty, \cdot)=0, u(+\infty, \cdot)=1
\end{array}\right.
$$

In short, this problem can be motivated by deflagration curved flame propagation models in the theory of combustion and by biological situations. The first works in this multidimensional case were in particular made by Berestycki, Larrouturou, Lions and Nirenberg ( $c f$. [4], [5], [7]). These authors have especially generalized some known results on the ordinary differential equation $u^{\prime \prime}-c u^{\prime}+f(u)=0$ with the boundary conditions $u(-\infty)=0$ and $u(+\infty)=1$. This last situation corresponds to the propagation of planar waves and the works about it were initiated by Kolmogorov-Petrovskii-Piskunov, Zeldovic-Frank-Kamenetskii, Kanel' and Fife-McLeod ( $c f$. [9], [12], [13], [25]).

In the literature, one usually has to distinguish three main cases:

- case A: $\exists \theta \in(0,1)$ such that $f \equiv 0$ on $[0, \theta], f>0$ on $(\theta, 1)$ ( $\theta$ is an "ignition temperature", see the explanations below), $f(1)=0$.

- case B: $\exists \theta \in(0,1)$ such that $f<0$ on $(0, \theta), f(\theta)=0$ and $f>0$ on $(\theta, 1), f(0)=f(\theta)=f(1)=0$ ("bistable case").

- case C: $f>0$ on $(0,1), f(0)=f(1)=0$ (Fisher, or "KPP" type).

Case A ("ignition temperature") is motivated by the theory of combustion. Roughly speaking, the starting point is the thermo-diffusive model for wrinkled deflagration flame propagation in an infinite tube where a simple chemical reaction $\mathcal{A} \rightarrow \mathcal{B}$ takes place between two premixed gases. The function $u$ is the renormalized temperature of the mixture and $1-u$ is the renormalized concentration of the reactant $\mathcal{A}$ (see the synthetic works of Berestycki, Larrouturou, Sivashinsky and Williams [3], [16], [20]). Explicitely, in models of combustion, the real $\theta$ represents an ignition temperature below which no reaction happens. The source term $f$ takes into account the mass action law and Arrhenius's law. The convection term $\beta(y, c) \partial_{1} u$ is often of the form $(c+\alpha(y)) \partial_{1} u$ (or sometimes $c \alpha(y) \partial_{1} u$, $\alpha>0$ on $\bar{\omega})$. The function $\alpha$ is a mass flow distribution given on $\bar{\omega}$ and is uniform along the principal direction of the cylinder. If the coefficient of the convection term is of the form $\beta(y, c)=c+\alpha(y)$, then the functions $u$ solutions of $\left(P_{\text {inv }}\right)$ are travelling front solutions for the following evolution problem of reaction-diffusion $\partial_{t} U=\Delta U-\alpha(y) \partial_{1} U+f(U)$. In other words, $c$ represents the speed of a front of the flame and $u$ its profile. 
Case B ("bistable") mainly occurs in biological situations: the function $u$ represents for instance the concentration of some species ( $c f$. [2], [9]).

Case C ("KPP") corresponds as well to biological phenomena as combustion models when the ignition temperature vanishes ( $c f .[13],[25]$ ).

Anyway, the boundary condition $\partial_{\nu} u=0$ on $\partial \Sigma$ means that there is no flow across the walls of the cylinder. The limits $u(-\infty, \cdot)=0$ and $u(+\infty, \cdot)=1$ mean that the flame moves with speed $c$ from the burnt gases in $+\infty$ to the fresh zone in $-\infty$.

If one moreover assumes that $f^{\prime}(1)$ exists and is $<0$, and $f$ is of class $C^{1, \delta}$ near 0 to the right and 1 to the left for some $0<\delta<1$, the main results for problem $\left(P_{\text {inv }}\right)$ can be summarized as follows:

THEOREM 0 ([7]). - In case A ("ignition temperature"), there exists a pair $(c, u)$ solution of $\left(P_{i n v}\right)$. The real $c$ is unique and the function $u$ is unique up to translation with respect to $x_{1}$.

In case $B$ ("bistable case"), if $f^{\prime}(0)<0, \omega$ is convex and if $f$ is of class $C^{1, \delta}([0,1])$ for some $\delta>0$, the same result holds.

In case C ("KPP" case), if $f^{\prime}(0)>0$, there exists a minimal speed $c^{*}$ and solutions $(c, u)$ of $\left(P_{i n v}\right)$ if and only if $c \geq c^{*} ;$ for any $c \geq c^{*}$, these solutions $u$ are unique up to translation in the $x_{1}$-direction.

Similar problems were studied by Xin ( $c f .[21])$ in periodic media $\mathbb{R} \times T$, where $T$ is the unit torus in $\mathbb{R}^{N}$. In [21] and [22], Xin proved existence, uniqueness and monotonicity results, using fixed point theorem, continuation and sliding methods. In the case where $f$ is of "bistable" type, Xin also studied, in periodic media, some non homogeneous problems for which the diffusion term $\Delta u$ is replaced by an approximated expression $\operatorname{div}((I+A) \nabla u)$ where $A(x)$ is a matrix defined in a periodic box of $\mathbb{R}^{N}$. Xin proved the existence of solutions for small $A(c f .[23])$ and non existence for $A$ with large enough variation ([24]).

Volpert and Volpert also studied a dependence on $x_{1}$ in the source term $f\left(x_{1}, u\right)$ for systems of ordinary differential equations, $u$ representing a vector of functions. They proved the existence of monotone solutions and a priori estimates for such solutions. In this paper, we actually generalize in the multidimensional case some results given in $|19|$. and we even show that the solutions of $(P)$ are increasing in $x_{1}$.

We now come back to problem $(P)$ constituted of the equation $(E)$ and the boundary conditions $(B . C)$ ). Many questions remained open about problems set in unhomogeneous media. The difference between the case of a velocity field $\beta=\beta(y, c)$ and the situation under examination with 
an additional dependence on $x_{1}$ in this velocity field and in the source term means that the medium is fully non homogeneous along the principal direction of the cylinder. For the thermodiffusive model, this can mean that we do not assume the constant density approximation; physically, the hydrodynamical effects due to the heat expansion have a non negligible function with respect to the reaction phenomena (see [14]).

Some perturbations in the medium may occur in a localized zone around the front of the wave in the frame which moves with speed $c$ to the left. We refer to [10] for the case of a non homogeneous convection term of the form $(c+\alpha(y)) \partial_{1} u+\vec{q} \cdot \nabla u$ where $\vec{q}$ is a small perturbation of the velocity distribution which is in $L^{\infty}(\mathbb{R})$; this can be interpreted as a first step in the introduction of turbulence.

The physical problem of a non constant velocity field $\beta\left(x_{1}, y, c\right)$ can remind us of the flow in a plane engine, where the velocities of the gases in the entrance and in the exit are different.

In all what follows, according to the brief physical explanations above, we will assume to simplify that $\beta$ is non increasing in $x_{1}$ and $f$ is non decreasing in $x_{1}$. The function $f$ may for instance be of the type $f\left(x_{1}, u\right)=\tilde{f}(u) \psi\left(x_{1}\right)$ where $\tilde{f}$ is a positive function and $\psi$ is an increasing function bounded from below by a positive constant on $\mathbb{R}$. The mathematical stake is to understand the difference between the invariant by translation problem $\left(P_{i n v}\right)$ and the non invariant one $(P)$. Indeed, the first important remark is the following: if $x_{0} \in \mathbb{R}$ and $(c, u)$ is a solution of $(P)$, then the translated function $\left(x_{1}, y\right) \mapsto u\left(x_{1}+x_{0}, y\right)$ is not necessarly solution of $(P)$ with this speed $c$. This is in sharp contrast with the solutions of problem $\left(P_{i n v}\right)$.

In the theory of ordinary differential equations, we know the difference between the equations with constant coefficients and the equations with non constant coefficients. In a similar way, the aim of this article is to describe some results for problem $(P)$ when the coefficients $\beta$ and $f$ depend on $x_{1}$, but only in the monotone case indicated above. These results will explicitely clarify the qualitative difference with respect to the invariant by translation situation $\left(P_{\text {inv }}\right)$.

\section{Main results}

Shortly, we study the structure of the set of solutions of problem $(P)$, and we establish the connection with two asymptotic problems, which correspond to the limits $x_{1} \rightarrow \pm \infty$, which we note $\left(P_{ \pm}\right)$, the "limit 
problems" in $\pm \infty$, and which are invariant by $x_{1}$-translation,

$$
\left\{\begin{array}{c}
\Delta u-\beta_{ \pm}(y, c) \partial_{1} u+f_{ \pm}(u)=0 \text { in } \Sigma \\
\partial_{\nu} u=0 \text { on } \partial \Sigma \\
u(-\infty, \cdot)=0, u(+\infty, \cdot)=1
\end{array}\right.
$$

Apart the general assumptions made on the functions $\beta$ and $f$, we will consider that $f_{+}$and $f_{-}$satisfy one of the three main cases indicated above: "ignition case" A, "bistable" case B and "ZFK" case C. We mention that in all cases $f_{-} \leq f_{+}$because $f$ is non decreasing in $x_{1}$, and $\beta_{-}(y, c) \geq \beta_{+}(y, c)$ because $\beta$ is non increasing in $x_{1}$. More precisely, one will investigate three situations concerning the profiles of the functions $f_{-}$and $f_{+}$:

Case I: $f_{-}$and $f_{+}$satisfy the "ignition temperature" case $\mathrm{A}$, with respective ignition temperatures $\theta_{-}$and $\theta_{+}$such that $0<\theta_{+} \leq \theta_{-}<1$. It is furthermore assumed that $f_{-}$and $f_{+}$are of class $C^{1, \delta}$ near 1 , for some $\delta>0$, and $f_{-}^{\prime}(1), f_{+}^{\prime}(1)<0, f_{+}^{\prime}\left(\theta_{+}\right)>0$.

Case II: $f_{-}$and $f_{+}$satisfy the "bistable" case $\mathrm{B}$, with respective zeros $\theta_{-}$and $\theta_{+}$such that $0<\theta_{+} \leq \theta_{-}<1$. It is assumed that $f_{-}^{\prime}(0), f_{+}^{\prime}(0)$, $f_{-}^{\prime}(1), f_{+}^{\prime}(1)<0$. Moreover, one assumes that the section $\omega$ is convex. The functions $f_{-}, f_{+}$are of class $C^{1, \delta}$ with respect to $u$ for some $\delta>0$ and $f_{-}^{\prime}\left(\theta_{-}\right)>0, f_{+}^{\prime}\left(\theta_{+}\right)>0$.

Case III: $f_{-}$and $f_{+}$satisfy "KPP" case $\mathrm{C}$ and $f_{-}^{\prime}(0), f_{+}^{\prime}(0)>0, f_{-}^{\prime}(1)$, $f_{+}^{\prime}(1)<0$.

From the results recalled above in theorem 0 , in both cases I and II, there exist some unique pairs $\left(c_{-}, u_{-}\right)$and $\left(c_{+}, u_{+}\right)$solutions of the limit problems $\left(P_{-}\right)$and $\left(P_{+}\right)\left(u_{ \pm}\right.$are unique up to translation in the $x_{1}$ direction). In case III, there exist two minimal speeds $c_{-}^{*}$ and $c_{+}^{*}$ and solutions $(c, u)$ for problems $\left(P_{-}\right)$and $\left(P_{+}\right)$if and only if $c \geq c_{-}^{*}$ and $c \geq c_{+}^{*}$.

We can now state the different results for the existence of solutions of the initial non invariant problem $(P)$, which are summarized in the following theorem:

THEOREM 1 (Existence)

a) In cases I and II ( $f_{ \pm}$are of "ignition temperature" or "bistable" types), $f$ being non decreasing in $x_{1}$ and $\beta$ non increasing in $x_{1}$, then $c_{-} \leq c_{+}$. Moreover, in each of the following cases, we have $c_{-}<c_{+}$:

$$
-\beta_{-}(\cdot, c) \not \equiv \beta_{+}(\cdot, c) \forall c \in \mathbb{R}
$$


- in case II, $f_{-}^{\prime}(0)<f_{+}^{\prime}(0)<0$ and $f_{+}^{\prime}(1)<f_{-}^{\prime}(1)<0$.

$-f_{-} \not \equiv f_{+}$and $\left|f_{-}(1-s)-f_{+}(1-s)\right|=O\left(s^{1+\delta}\right)$ as $s \rightarrow 0$, for some $\delta>0$.

- in case $I, f_{+}^{\prime}(1)<f_{-}^{\prime}(1)<0$.

Furthermore, if $c_{-}<c_{+}$, then for any $c_{-}<c<c_{+}$, there exists a solution $(c, u)$ of $(P)$ such that $\partial_{1} u \geq 0$ in $\bar{\Sigma}$.

If $c_{-}=c_{+}$, then there exists a solution $u$ such that $\partial_{1} u \geq 0$ in $\bar{\Sigma}$ with the speed $c=c_{-}=c_{+}$.

b) In case III ( $f_{ \pm}$are of "KPP" type $), f$ being non decreasing in $x_{1}$ and $\beta$ non increasing in $x_{1}$, then $c_{-}^{*} \leq c_{+}^{*}$. For any $c \geq c_{+}^{*}$ and for any $h \in(0,1)$, there exists a solution $(c, u)$ of $(P)$ such that $\partial_{1} u \geq 0$ in $\bar{\Sigma}$ and $\max _{\bar{\omega}} u(0, \cdot)=h$.

Remarks. - Since $f_{-}$and $f_{+}$are of class $C^{1, \delta}$ near 1 , if $f_{-}^{\prime}(1)=f_{+}^{\prime}(1)$, then the assumption $\left|f_{-}(1-s)-f_{+}(1-s)\right|=O\left(s^{1+\delta}\right)$ is automatically satisfied.

In the case of a velocity field $\beta\left(x_{1}, y, c\right)=c+\alpha(y)+\gamma\left(x_{1}\right)$ where $\gamma$ is a non increasing function on $\mathbb{R}$, then the case $c_{-}=c_{+}$only occurs if and only if $\gamma$ and $f$ are invariant in $x_{1}$; otherwise $c_{-}<c_{+}$.

The second main part of this article is devoted to the precise study of the set of the solutions of problem $(P)$ in the different cases I, II and III, and to state some a priori properties of such solutions. We begin to state some $a$ priori conditions on the speeds $c$ solutions.

THEOREM 2. - The function $f$ being as usual non decreasing in $x_{1}$ and $\beta$ non increasing in $x_{1}$, in cases I and II ( $f_{ \pm}$being of "ignition temperature" or "bistable" types), if $(c, u)$ is a solution of $(P)$ such that $\partial_{1} u \geq 0$, then $c_{-} \leq c \leq c_{+}$. In case III ( $f_{ \pm}$are of "KPP" type $)$, if $\partial_{1} u \geq 0$, then $c \geq c_{-}^{*}$.

The next two theorems concern the properties of the eventual functions $u$ solutions of $(P)$. For that purpose, we will assume some technical hypotheses on the regularity of the functions $f$ and $\beta$ and on their behaviours as $x_{1} \rightarrow \pm \infty$. At first, $f$ and $\beta$ are assumed to be of class $C^{1}$ with respect to $x_{1}$. Besides, they tend exponentially to $f_{ \pm}$and $\beta_{ \pm}$as $x_{1} \rightarrow \pm \infty$ :

$\left\{\begin{array}{cc}\forall \alpha>0 & \left|f\left(x_{1}, u\right)-f_{+}(u)\right|=O\left(e^{-\alpha x_{1}}\right) \text { as } x_{1} \rightarrow+\infty \\ \forall \alpha>0 & \left|f\left(x_{1}, u\right)-f_{-}(u)\right|=O\left(e^{-\alpha\left|x_{1}\right|}\right) \text { as } x_{1} \rightarrow-\infty\end{array}\right.$ uniformly in $u$ and $\exists C_{ \pm}, \delta_{ \pm}>0$ such that

$$
\left\{\begin{array}{l}
\left|\beta\left(x_{1}, y, c\right)-\beta_{-}(y, c)\right| \leq C_{-} e^{\delta_{-} x_{1}} \quad \forall x_{1} \leq 0, y \in \bar{\omega}, c \in \mathbb{R} \\
\left|\beta\left(x_{1}, y, c\right)-\beta_{+}(y, c)\right| \leq C_{+} e^{-\delta_{+} x_{1}} \quad \forall x_{1} \geq 0, y \in \bar{\omega}, c \in \mathbb{R}
\end{array}\right.
$$


(if $\beta\left(x_{1}, y, c\right)=c+\alpha(y)+\gamma\left(x_{1}\right)$, these assertions mean that $\left|\gamma-\gamma_{ \pm}\right|$tend faster than some exponential as $x_{1} \rightarrow \pm \infty$ ). Moreover, these assertions are satisfied if $\partial_{x_{1}} \beta=0$ for $\left|x_{1}\right|$ large enough.

We split the results on the structure of the solutions of problem $(P)$ into two main theorems:

THFORFM 3. - With the assumptions above on the behaviour of $f$ and $\beta$ as $x_{1} \rightarrow \pm \infty$, $f$ being as usual non decreasing in $x_{1}$ and $\beta$ non increasing in $x_{1}$, if $(c, u)$ is a solution of $(P)$ in cases $I, I I$ and III, then $\partial_{1} u>0$ in $\bar{\Sigma}$.

Remark 1. - Under the above hypotheses, it follows from theorems 2 and 3 that one has $c_{-}<c<c_{+}$in cases I and II, and $c>c_{-}^{*}$ in case III.

Remark 2. - Theorems 2 and 3 yield that the existence results given in theorem 1 in cases I and II are quite optimal, up to the existence in the limit cases $c=c_{-}$or $c=c_{+}$.

THEOREM 4. - With the assumptions above on the behaviour of $f$ and $\beta$ as $x_{1} \rightarrow \pm \infty, f$ being as usual non decreasing in $x_{1}$ and $\beta$ non increasing in $x_{1}$; in both cases I and II ( $f_{ \pm}$are of "ignition temperature" or "bistable" types), we have

a) if $(c, u)$ and $\left(c^{\prime}, u^{\prime}\right)$ are solutions of $(P)$ such that $c<c^{\prime}$, then $u>u^{\prime}$ in $\bar{\Sigma}$.

b) if one moreover assumes that $\beta\left(x_{1}, y, c\right)=\beta_{+}(y, c)$ for $x_{1}$ large enough, uniformly in $(y, c)$, let $c_{-} \leq c \leq c_{+}$and fix $0<h<1$, then there exists at most one solution of $(P)$ which satisfies the normalization condition $\max _{\bar{\omega}} u(0, \cdot)=h$.

In a few words, the existence theorem can be proved by a passage to the limit in finite cylinders, for which the existence of solutions is given by a result on sub and super-solutions. The comparison with auxiliary functions is needed to obtain the limit conditions as $x_{1} \rightarrow \pm \infty$. The main tools for the necessary conditions are based on the study of the exponential behaviours of the different solutions, some results given in the appendix are useful.

\section{EXISTENCE RESULTS, PROOF OF THEOREM 1}

\subsection{Cases I and II ("ignition temperature" and "bistable" cases)}

\subsubsection{Comparison between $c_{-}$and $c_{+}$}

The demonstration of the inequality $c_{-} \leq c_{+}$can be made by contradiction and is based on the study of the exponential decays of 
solutions. This allows to begin a sliding method. Similar proofs were made in [7].

Let us first suppose that $c_{-}>c_{+}$and argue by contradiction. Let us study the exponential behaviours of $u_{-}$and $u_{+}$near $\pm \infty$ and for that purpose apply the results of [7] $(\S 2,3,4)$.

We first consider case I. Since $\beta_{-}\left(\cdot, c_{-}\right)>\beta_{+}\left(\cdot, c_{+}\right)$(because $c_{-}>c_{+}$ and $\beta$ is non increasing in $x_{1}$ ), near $-\infty$, the functions $u_{-}$and $u_{+}$satisfy

$$
u_{\perp}\left(x_{1}, y\right)=e^{\lambda_{ \pm} x_{1}} \phi_{ \pm}(y)+o\left(e^{\lambda_{ \pm} x_{1}}\right) \text { as } x_{1} \rightarrow-\infty \text { uniformly in } y \in \bar{\omega}
$$

with two reals $0<\lambda_{+}<\lambda_{-}$and two functions $\phi_{ \pm}>0$ on $\bar{\omega}$ solutions of

$$
\left\{\begin{array}{c}
\Delta \phi_{ \pm}+\left(\lambda_{ \pm}^{2}-\lambda_{ \pm} \beta_{ \pm}\left(y, c_{ \pm}\right)\right) \phi_{ \pm}=0 \text { in } \omega \\
\partial_{\nu} \phi_{ \pm}=0 \text { on } \partial \omega
\end{array}\right.
$$

It comes that $0<u_{-}<u_{+}$near $-\infty$.

On the other side, one necessarly has $f_{+}^{\prime}(1) \leq f_{-}^{\prime}(1)<0$ and $\beta_{-}\left(\cdot, c_{-}\right)>\beta_{+}\left(\cdot, c_{+}\right)$. Thus, from the results of $[7]$, one can write

$u_{ \pm}\left(x_{1}, y\right)=1-e^{\mu_{ \pm} x_{1}} \psi_{ \pm}(y)+o\left(e^{\mu_{ \pm} x_{1}}\right)$ as $x_{1} \rightarrow+\infty$ uniformly in $y \in \bar{\omega}$ with $\mu_{+}<\mu_{-}<0$ and $\psi_{ \pm}>0$ solutions of

$$
\left\{\begin{array}{c}
\Delta \psi_{ \pm}+\left(\mu_{ \pm}^{2}-\mu_{ \pm} \beta_{ \pm}\left(y, c_{ \pm}\right)+f_{ \pm}^{\prime}(1)\right) \psi_{ \pm}=0 \text { in } \omega \\
\partial_{\nu} \psi_{ \pm}=0 \text { on } \partial \omega
\end{array}\right.
$$

Indeed, in order to explain this, let us recall that $\mu_{ \pm}<0$ are solutions of

$$
\mu_{ \pm}^{2}=\mu_{1}\left(-\Delta+\mu_{ \pm} \beta_{ \pm}\left(y, c_{ \pm}\right)-f_{ \pm}^{\prime}(1)\right)
$$

$\mu_{1}(-L)$ denoting the principal eigenvalue of the elliptic operator $L$ with Neumann boundary conditions. The functions $g_{ \pm}: t \mapsto \mu_{1}(-\Delta+$ $\left.t \beta_{ \pm}\left(y, c_{ \pm}\right)-f_{ \pm}^{\prime}(1)\right)$ are strictly decreasing with $\beta$ (considered as a variable) for each $t<0$, and $g_{+}(0)=-f_{+}^{\prime}(1) \geq-f_{-}^{\prime}(1)=g_{-}(0)$. Hence, since $c_{+}<c_{-}$, which yields $\beta_{+}\left(\cdot, c_{+}\right)<\beta_{-}\left(\cdot, c_{-}\right)$, we have $g_{+}(t)>g_{-}(t)$ for any $t<0$, and thus

$$
\mu_{+}<\mu_{-}<0
$$

It comes then that $u_{-}<u_{+}<1$ in the neighbourhood of $+\infty$.

We now use a sliding method: at first there exists $R>0$ such that $u_{-}<u_{+}$if $\left|x_{1}\right| \geq R, y \in \bar{\omega}$. As $u_{-}$is increasing with $x_{1}$ and $u_{+}$is greater than some $\alpha>0$ on the compact set $[-R, R] \times \bar{\omega}$, we can translate 
$u_{-}$to the left enough such that $u_{-}\left(x_{1}-s, y\right)<u_{+}\left(x_{1}, y\right)$ for some $s>0$ and for any $\left(x_{1}, y\right) \in \bar{\Sigma}$. As the behaviours of $u_{+}$and $u_{-}$are exponentially different in $\pm \infty$, we can actually translate $u_{-}\left(x_{1}-s, y\right)$ to the right in such a way that for some $t \leq s$

$$
u_{-}\left(x_{1}-t, y\right) \leq u_{+}\left(x_{1}, y\right) \text { in } \bar{\Sigma}
$$

with equality somewhere in $\bar{\Sigma}$. Thus, the function $z=u_{+}\left(x_{1}, y\right)-u_{-}\left(x_{1}-\right.$ $t, y)$ is $\geq 0$ and $=0$ somewhere, it satisfies

$$
\left\{\begin{array}{c}
\Delta z-\beta_{-}\left(y, c_{-}\right) \partial_{1} z+\left(f_{+}\left(u_{+}\left(x_{1}, y\right)\right)-f_{+}\left(u_{-}\left(x_{1}-t, y\right)\right)\right) \\
=\left(-\beta_{-}\left(y, c_{-}\right)+\beta_{+}\left(y, c_{+}\right)\right) \partial_{1} u_{+} \\
+\left(f_{-}\left(u_{-}\left(x_{1}-t, y\right)\right)-f_{+}\left(u_{-}\left(x_{1}-t, y\right)\right)\right) \text { in } \Sigma \\
\partial_{\nu} z=0 \text { on } \partial \Sigma
\end{array}\right.
$$

We have already infered that $\beta_{-}\left(\cdot, c_{-}\right)>\beta_{+}\left(\cdot, c_{+}\right)$. Moreover, $\partial_{1} u_{+} \geq 0$ and $f_{-} \leq f_{+}$. Lastly, as $f_{+}$is lipschitz-continuous, there exists a function $c \in L^{\infty}(\bar{\Sigma})$ such that

$$
\left\{\begin{array}{c}
\Delta z-\beta_{-}\left(y, c_{-}\right) \partial_{1} z+c(x) z \leq 0 \text { in } \Sigma \\
\partial_{\nu} z=0 \text { on } \partial \Sigma
\end{array}\right.
$$

Finally, the maximum principle and Hopf lemma yield that $z \equiv 0$ in $\bar{\Sigma}$, which is a contradiction with the exponential behaviours of $u_{-}$or $u_{+}$near $\pm \infty$. That proves the first assertion in theorem 1 , in case I.

In case II, in order to obtain $c_{-} \leq c_{+}$, the proof is similar, the estimates in $-\infty$ can be treated in the same way as in $+\infty$.

In cases $\mathrm{I}$ and II, if we add the following hypothesis: $\beta_{-}(y, c) \geq \beta_{+}(y, c)$ for all $c$, applying the results of [7], then we can prove exactly in the same way that the assumption $c_{-} \leq c_{+}$would yield a contradiction. One can remark that this situation occurs if $\beta\left(x_{1}, y, c\right)=c+\alpha(y)+\gamma\left(x_{1}\right)$ with a function $\gamma$ non increasing and non constant.

In case II, the hypotheses $f_{-}^{\prime}(0)<f_{+}^{\prime}(0)<0$ and $f_{+}^{\prime}(1)<f_{-}^{\prime}(1)<0$ allow to obtain a contradiction if $c_{-}=c_{+}$, with the same arguments: indeed, even if $c_{-}=c_{+}$, the exponential behaviours of $u_{-}$and $u_{+}$can be compared near $\pm \infty$.

If $f_{-} \not \equiv f_{+}$and $\left|f_{-}(1-s)-f_{+}(1-s)\right|=O\left(s^{1+\delta}\right)$ as $s \rightarrow 0$, for some $\delta>0$, then we also have $c_{-}<c_{+}$. The proof is quite technical and will be given in section 3.3.3. Indeed, it corresponds to a similar situation as the one developped in section 3.3.3. Case I, with $f_{+}^{\prime}(1)<f_{-}^{\prime}(1)<0$, is similar. 
Remark. - To sum up, since $f_{-}$and $f_{+}$are of class $C^{1, \delta}$ near 1 , then $c_{-}=c_{+}$only if $f_{-} \equiv f_{+}$, i.e. $f\left(x_{1}, u\right)=f(u)$ and $\exists c$ such that $\beta_{-}(\cdot, c) \equiv \beta_{+}(\cdot, c)$.

\subsubsection{Existence of a solution for any $c_{-}<c<c_{+}$}

We proved in the previous section that $c_{-} \leq c_{+}$and explicited sufficient conditions to have $c_{-}<c_{+}$In this part, we assume that $c_{-}<c_{+}$. Our purpose is to adapt the methods of Volpert and Volpert ( $c f$. [19]). The basic idea is to use an argument of sub and super-solutions. At first, we will construct some auxiliary solutions in semi-infinite cylinders.

a) Construction of auxiliary solutions in semi-infinite cylinders

We recall that we consider the cases of functions $f_{ \pm}$which are of "ignition temperature" or "bistable" types. Following the ideas of [9], for any fixed $c<c_{+}$, we will construct a function $w$ defined in $\mathbb{R}_{+} \times \bar{\omega}$ solution of

$$
\left\{\begin{array}{c}
\Delta w-\beta_{+}(y, c) \partial_{1} w+f_{+}(w)=0 \text { in } \Sigma_{+}=\mathbb{R}_{+} \times \omega \\
w(0, \cdot)=0 \\
w(+\infty, \cdot)=1 \\
\partial_{\nu} w=0 \text { on } \mathbb{R}_{+} \times \partial \omega
\end{array}\right.
$$

and $\partial_{1} w \geq 0$ in $\overline{\Sigma_{+}}$.

We will at first solve the problem in finite cylinders $R_{a}=(0,2 a) \times \omega$. The method of sub- and super-solutions developped in [8] (Th. 7.2) yields that there exists a function $u_{a}$ such that

$$
\left\{\begin{array}{c}
\Delta u_{a}-\beta_{+}(y, c) \partial_{1} u_{a}+f_{+}\left(u_{a}\right)=0 \text { in } R_{a} \\
\partial_{\nu} u_{a}=0 \text { on }(0,2 a) \times \partial \omega \\
u_{a}(0, \cdot)=0, u_{a}(2 a, \cdot)=1
\end{array}\right.
$$

This solution $u_{a}$ also satisfies $\partial_{1} u_{a} \geq 0$ in $R_{a}$. Using standard local estimates up to the boundary and the Sobolev injections, we deduce that for a subsequence $a \rightarrow+\infty$, the functions $u_{a}$ tend to a function $w$ in $C_{\text {loc }}^{1, \mu}\left(\overline{\Sigma_{+}}\right)(\mu>0)$. The limit function $w$ satisfies:

$$
\left\{\begin{array}{c}
\Delta w-\beta_{+}(y, c) \partial_{1} w+f_{+}(w)=0 \text { in } \Sigma_{+} \\
\partial_{\nu} w=0 \text { on } \mathbb{R}_{+}^{*} \times \partial \omega \\
w(0, \cdot)=0, w(+\infty, y)=\psi(y)
\end{array}\right.
$$

where $\psi \in W^{2, p}(\omega)$ is a solution of the stationnary problem

$$
\left\{\begin{array}{c}
\Delta \psi+f_{+}(\psi)=0 \text { in } \omega \\
\partial_{\nu} \psi=0 \text { on } \partial \omega
\end{array}\right.
$$


We want to prove that $\psi \equiv 1$. Arguing by contradiction, let us suppose that $\psi \neq \equiv 1$. Since $0 \leq \psi \leq 1$, it follows from the maximum principle and Hopf lemma that $0 \leq \psi<1$ in $\bar{\omega}$.

Let us fix a real number $d<1$ such that $\max \left(\theta_{+}, \max _{\bar{\omega}} \psi\right)<d<1$. Since $u_{a}$ is increasing in $x_{1}$, there exists a real $\tau_{a} \in(0,2 a)$ such that $\min _{\bar{\omega}} u_{a}\left(\tau_{a}, \cdot\right)=d$. As $u_{a} \rightarrow w$ locally and $w \leq \psi$, it comes that $\tau_{a} \rightarrow+\infty$ as $a \rightarrow+\infty$.

Let us now shift the origin to $x_{1}=\tau_{a}$ by setting $v_{a}\left(x_{1}, y\right)=$ $u_{a}\left(x_{1}+\tau_{a}, y\right)$ in the cylinder $\left[-\tau_{a}, 2 a-\tau_{a}\right] \times \bar{\omega}$. For a sub-sequence $a \rightarrow \infty$, we have $2 a-\tau_{a} \rightarrow b \in[0,+\infty]$, and the family $\left(v_{a}\right)$ converges to a function $v_{c}$ locally in $\left.\left.C^{1, \mu}(]-\infty, b\right] \times \bar{\omega}\right)$, which satisfies the same equation as $w$. Moreover, $\min _{\bar{\omega}} v_{c}(0, \cdot)=d$ and $v_{c}$ has a limit in $-\infty$ $\left(v_{c}(-\infty, y)=\psi_{1}(y)\right)$ where $\psi_{1}$ is a solution of $(2.1)$ such that $\psi_{1} \geq \psi$ since $\tau_{a} \rightarrow+\infty$ and $u_{a}$ is increasing in $x_{1}$. Two cases may a priori occur: i) $b \in[0,+\infty[$ and ii) $b=+\infty$. In each of these cases, we will obtain a contradiction by a sliding method:

in case i), $v_{c}(b, \cdot)=1$. If $\psi_{1} \equiv 0$, as in the previous sections, one can compare the exponential behaviours of $v_{c}$ and $u_{+}$in $-\infty$ and, using the hypothesis $c<c_{+}$, one would obtain a contradiction after a sliding method. Otherwise, from the maximum principle and Hopf lemma, one has $\psi_{1}>0$, and one actually concludes in the same way.

In case ii), $v_{c}(+\infty, y)=\psi_{2}(y)$. The function $\psi_{2}$ satisifes $(2.1)$ and $\min \psi_{2} \geq d>\theta_{+}$since $v_{c}$ is increasing in $x_{1}$ and from the normalization condition on $\{0\} \times \bar{\omega}$. By integration of (2.1) in $\omega$, and since $f_{+}>0$ on $\left(\theta_{+}, 1\right)$, we conclude that $\psi_{2} \equiv 1$. As above, we would finally obtain a contradiction by a sliding method.

For any fixed $c>c_{-}$, in case II, we can argue exactly in the same way to prove the existence of a function $w$ such that

$$
\left\{\begin{array}{c}
\Delta w-\beta_{-}(y, c) \partial_{1} w+f_{-}(w)=0 \text { in } \Sigma_{-}=\mathbb{R}_{-} \times \omega \\
\partial_{\nu} w=0 \text { on } \mathbb{R}_{-}^{*} \times \partial \omega \\
w(-\infty, \cdot)=0, w(0, \cdot)=1
\end{array}\right.
$$

and $\partial_{1} w \geq 0$ in $\Sigma_{-}$.

In case $\mathrm{I}$, there is just a new argument to add because $f_{-} \equiv 0$ on $\left[0, \theta_{-}\right]$. One constructs functions $u_{a}$ in $[-2 a, 0] \times \bar{\omega}$ solutions of

$$
\left\{\begin{array}{c}
\Delta u_{a}-\beta_{-}(y, c) \partial_{1} u_{a}+f_{-}\left(u_{a}\right)=0 \text { in }(-2 a, 0) \times \omega \\
u_{a}(-2 a, \cdot)=0, u_{a}(0, \cdot)=1 \\
\partial_{\nu} u_{a}=0 \text { on }(-2 a, 0) \times \partial \omega
\end{array}\right.
$$


and then pass to the limit for a sequence $a \rightarrow+\infty$. The limit function $w$ satisfies the equations

$$
\left\{\begin{array}{c}
\Delta w-\beta_{-}(y, c) \partial_{1} w+f_{-}(w)=0 \text { in } \Sigma_{-} \\
\partial_{\nu} w=0 \text { on } \mathbb{R}_{-}^{*} \times \partial \omega \\
w(-\infty, y)=\psi(y), w(0, \cdot)=1
\end{array}\right.
$$

One wants to prove that $\psi \equiv 0$ on $\bar{\omega}$. In the other case, $\psi>0$ on $\bar{\omega}$ and one introduces a real $d>0$ such that $d<\min \left(\min \psi, \theta_{-}\right)$and $\tau_{a}$ such that $\max _{\bar{\omega}} u_{a}\left(-\tau_{a}, \cdot\right)=d$. Since $\partial_{1} w \geq 0$ and $w(-\infty, y)=\psi(y)$, one obtains that $\tau_{a} \rightarrow+\infty$. One defines the translated functions $v_{a}=u_{a}\left(-\tau_{a}+x_{1}, y\right)$ in $\left[-2 a+\tau_{a}, \tau_{a}\right] \times \bar{\omega}$. They converge to a function $v_{c}$ in $[b,+\infty[\times \omega$ in suitable spaces. Since $c>c_{-}$, it comes that $\int_{\omega} \beta_{-}\left(y, c_{-}\right)>0$ (by integration of the equation satisfied by $u_{-}$). From the results of [7], there exists an exponential function $z=e^{\lambda x_{1}} \phi(y)$ with $\lambda>0$ and $\phi>0$ on $\bar{\omega}$ solutions of

$$
\left\{\begin{array}{c}
\Delta z-\beta_{-}(y, c) \partial_{1} z=0 \text { in } \Sigma \\
\partial_{\nu} z=0 \text { on } \partial \Sigma
\end{array}\right.
$$

One can even assume that $\min \phi \geq \theta_{-}>d$. Since $v_{a} \leq d \leq \theta_{-}$in $\left[-2 a+\tau_{a}, 0\right] \times \bar{\omega}, f_{-}\left(v_{a}\right)=0$ in $\left[-2 a+\tau_{a}, 0\right] \times \bar{\omega}$. From the maximum principle and Hopf lemma, it comes that $v_{a} \leq z$ in $\left[-2 a+\tau_{a}, 0\right] \times \bar{\omega}$. Hence, by a passage to the limit $a \rightarrow+\infty$, one obtains

$$
v_{c}(-\infty, \cdot)=0 \text { if } b=-\infty
$$

One can then compare in the same way this function $v_{c}$ to $u_{-}$and obtain a contradiction. Obviously, the last step is similar if $b>-\infty$. This achieves the construction of these auxiliary functions $w$ defined in $\Sigma_{-}$or $\Sigma_{+}$.

b) Construction of a solution of $(P)$ for any $c_{-}<c<c_{+}$

In this part, one supposes that $c_{-}<c_{+}$and fix any $c$ in $\left(c_{-}, c_{+}\right)$. The proof of existence is devided in several main steps. Firstly, one constructs solutions in finite cylinders $[-a, a] \times \bar{\omega}$, and secondly one passes to the limit and concludes by comparison with the auxiliary solutions in semi-infinite cylinders.

STEP 1: Construction in finite cylinders and passage to the limit in infinite cylinders

Let $a>0$. We use the general results of [8] on sub- and super-solutions; there exists a solution $u_{a}$ defined in $[-a, a] \times \bar{\omega}$ of the following problem:

$$
\left\{\begin{array}{c}
\Delta u_{a}-\beta\left(x_{1}, y, c\right) \partial_{1} u_{a}+f\left(x_{1}, u_{a}\right)=0 \text { in }(-a, a) \times \omega \\
\partial_{\nu} u_{a}=0 \text { on }(-a, a) \times \partial \omega \\
u_{a}(-a, \cdot)=0, u_{a}(a, \cdot)=1
\end{array}\right.
$$

Vol. $14, \mathrm{n}^{\circ}$ 5-1997. 
because the constants 0 and 1 are respectively sub- and super-solutions for this problem. As $f$ is non decreasing in $x_{1}$ and $\beta$ non increasing in $x_{1}$, we can add that this solution is unique and such that $\partial_{1} u_{a}>0$ in $(-a, a) \times \bar{w}$. From the a priori elliptic estimates up to the boundary and the Sobolev injections, for some subsequence $a \rightarrow+\infty$, the functions $u_{a}$ tend to $u$ in $C_{l o c}^{1, \mu}(\bar{\Sigma})$. The function $u$ is solution of

$$
\left\{\begin{array}{c}
\Delta u-\beta\left(x_{1}, y, c\right) \partial_{1} u+f\left(x_{1}, u\right)=0 \text { in } \Sigma \\
\partial_{\nu} u=0 \text { on } \partial \Sigma
\end{array}\right.
$$

and is such that $\partial_{1} u \geq 0$ in $\bar{\Sigma}$.

STEP 2: Comparison with the auxiliary solutions as $x_{1} \rightarrow \pm \infty$

In case $I$, let $\chi_{ \pm}$two lipschitz-continuous functions defined on $[0,1]$ such that

$$
\left\{\begin{array}{l}
\chi_{ \pm} \equiv 0 \text { on }\left[0, \theta_{ \pm}\right] \\
\left.\left.\chi_{ \pm}>0 \text { on }\right] \theta_{ \pm}, 1\right]
\end{array}\right.
$$

and the restrictions of $\chi_{ \pm}$are assumed to be derivable on $\left[\theta_{ \pm}, 1\right]$ and $\chi_{ \pm}^{\prime} \geq \delta_{ \pm}>0$ on $\left[\theta_{ \pm}, 1\right]$. One introduces then the functions

$$
f_{ \pm}^{\epsilon}(u)=f_{ \pm}(u) \mp \epsilon \chi_{ \pm}(u)(1-u)
$$

For $\epsilon$ small enough, from the choice of the functions $\chi_{ \pm}$and since $f_{+}^{\prime}(1)<0, f_{+}^{\prime}\left(\theta_{+}\right)>0$, these functions $f_{ \pm}^{\epsilon}$ satisfy the hypotheses of theorem 0 in case A ("ignition temperature" case). Hence, there exist two unique pairs $\left(c_{ \pm}^{\epsilon}, u_{ \pm}^{\epsilon}\right)$ solutions of

$$
\left\{\begin{array}{c}
\Delta u_{ \pm}^{\epsilon}-\beta_{ \pm}\left(y, c_{ \pm}^{\epsilon}\right) \partial_{1} u_{ \pm}^{\epsilon}+f_{ \pm}^{\epsilon}\left(u_{ \pm}^{\epsilon}\right)=0 \text { in } \Sigma \\
\partial_{\nu} u_{ \pm}^{\epsilon}=0 \text { on } \partial \Sigma \\
u_{ \pm}^{\epsilon}(-\infty, \cdot)=0, u_{ \pm}^{\epsilon}(+\infty, \cdot)=1 \\
\max _{\bar{\omega}} u_{ \pm}^{\epsilon}(0, \cdot)=\theta_{ \pm}
\end{array}\right.
$$

Moreover, since $f_{ \pm}^{\epsilon}$ are close to $f_{ \pm}$, one can prove as in section 2.1.1 (comparison between $c_{-}$and $c_{+}$) that the speeds $c_{ \pm}^{\epsilon}$ are bounded. By a compactness argument, we obtain that the pairs $\left(c_{ \pm}^{\epsilon}, u_{ \pm}^{\epsilon}\right)$ converge as $\epsilon \rightarrow 0$ to a pair $\left(\tilde{c_{ \pm}}, \tilde{u_{ \pm}}\right)$. As in [7] $(\S 5)$, one can prove that the pairs $\left(\tilde{c_{ \pm}}, \tilde{u_{ \pm}}\right)$are actually solutions of the limit problems $\left(P_{ \pm}\right)$(one can identify the limits of the functions $\tilde{u_{ \pm}}$as being 0 and 1 in $\pm \infty$ by comparison with exponential functions). By uniqueness, one concludes that $\left(\tilde{c_{ \pm}}, \tilde{u_{ \pm}}\right)=\left(c_{ \pm}, u_{ \pm}\right)$.

In case $I I$, we define $f_{ \pm}^{\epsilon}(u)=f_{ \pm}(u) \mp \epsilon u(1-u)$. For $\epsilon$ small enough, the functions $f_{ \pm}^{\epsilon}$ satisfy the hypotheses of theorem 0 in case B (we use 
here the assumptions $f_{-}^{\prime}(0), f_{+}^{\prime}(1)<0$ and $\left.f_{-}^{\prime}\left(\theta_{-}\right), f_{+}^{\prime}\left(\theta_{+}\right)>0\right)$. In the same way, there exist unique pairs $\left(c_{ \pm}^{\epsilon}, u_{ \pm}^{\epsilon}\right)$ solutions of problems $\left(P_{ \pm}^{\epsilon}\right)$, and $\left(c_{ \pm}^{\epsilon}, u_{ \pm}^{\epsilon}\right) \rightarrow\left(c_{ \pm}, u_{ \pm}\right)$as $\epsilon \rightarrow 0$ (the demonstration is this of [7] $\S 5-6$ and uses the hypotheses of regularity of $f_{ \pm}$and the convexity of $\omega$ ).

One can now begin the main part of the demonstration. Since $c_{-}<c<$ $c_{+}$, there exist two reals $c^{\prime}$ and $c^{\prime \prime}$ and $\epsilon$ small enough such that

$$
c_{-}^{\epsilon}<c^{\prime}<c<c^{\prime \prime}<c_{+}^{\epsilon}
$$

From the results above, one introduces two functions $w_{ \pm}$solutions of

$$
\left\{\begin{array}{c}
\Delta w_{+}-\beta_{+}\left(y, c^{\prime \prime}\right) \partial_{1} w_{+}+f_{+}^{\epsilon}\left(w_{+}\right)=0 \text { in } \Sigma_{+} \\
w_{+}(0, \cdot)=0, w_{+}(+\infty, \cdot)=1 \\
\partial_{\nu} w_{+}=0 \text { on } \mathbb{P}_{+}^{*} \times \partial w
\end{array}\right.
$$

and

$$
\left\{\begin{array}{c}
\Delta w_{-}-\beta_{-}\left(y, c^{\prime}\right) \partial_{1} w_{-}+f_{-}^{\epsilon}\left(w_{-}\right)=0 \text { in } \Sigma_{-} \\
w_{-}(-\infty,)=0, w_{-}(0, \cdot)=1 \\
\partial_{\nu} w_{-}=0 \text { on } \mathbb{R}_{-}^{*} \times \partial \omega
\end{array}\right.
$$

Moreover, we recall that $\partial_{1} w_{-}, \partial_{1} w_{+} \geq 0$. We will compare the solutions $u_{a}$ (given by step 1) to some translated of $w_{-}$and $w_{+}$, for $-x_{1}$ and $x_{1}$ large enough respectively, in order to determine the limits of $u$ near $\pm \infty$.

In case II, one can write that

$$
\begin{aligned}
\Delta w_{+} & -\beta\left(x_{1}, y, c\right) \partial_{1} w_{+}+f\left(x_{1}, w_{+}\right) \\
= & f\left(x_{1}, w_{+}\right)-f_{+}\left(w_{+}\right)+\epsilon w_{+}\left(1-w_{+}\right) \\
& +\left(\beta_{+}\left(y, c^{\prime \prime}\right)-\beta\left(x_{1}, y, c\right)\right) \partial_{1} w_{+}
\end{aligned}
$$

Let us show that the second member is $\geq 0$ for $x_{1} \geq N$ large enough. Indeed, we can write

$$
\begin{aligned}
A & =f\left(x_{1}, w_{+}\right)-f_{+}\left(w_{+}\right)+\epsilon w_{+}\left(1-w_{+}\right) \\
& =\int_{0}^{w_{+}}\left(f_{u}^{\prime}\left(x_{1}, u\right)-f_{+}^{\prime}(u)\right) d u+\epsilon u_{1}\left(1-w_{+}\right)
\end{aligned}
$$

I.et us now fix $N_{1}$ such that $\left|f_{u}^{\prime}\left(x_{1}, u\right)-f_{+}^{\prime}(u)\right| \leq \epsilon / 4 \forall x_{1} \geq N_{1} \forall u \in[0,1]$. It is possible because we have assumed that $f_{u}^{\prime} \rightarrow f_{+}^{\prime}$ as $x_{1} \rightarrow+\infty$ uniformly in $u \in[0,1]$. If $x_{1} \geq N_{1}$ and $0 \leq w_{+} \leq 1 / 2$, then $A \geq-\epsilon / 4 w_{+}+\epsilon / 2 w_{+} \geq 0$. We can argue in the same way that $A \geq 0$ if $1 / 2 \leq w_{+} \leq 1$, writing $A=\int_{1}^{w_{+}}\left(f_{u}^{\prime}\left(x_{1}, u\right)-f_{+}^{\prime}(u)\right) d u+\epsilon w_{+}\left(1-w_{+}\right)$. 
In order to show that the second term $B=\left(\beta_{+}\left(y, c^{\prime \prime}\right)-\beta\left(x_{1}, y, c\right)\right) \partial_{1} w_{+}$ is $\geq 0$ for $x_{1}$ large enough, since $\partial_{1} w_{+} \geq 0$, it suffices to prove that $B^{\prime}=\beta_{+}\left(y, c^{\prime \prime}\right)-\beta\left(x_{1}, y, c\right)$ is $\geq 0$. We write

$$
B^{\prime}=\left(\beta_{+}\left(y, c^{\prime \prime}\right)-\beta_{+}(y, c)\right)+\left(\beta_{+}(y, c)-\beta\left(x_{1}, y, c\right)\right)
$$

From the hypothesis made on $\beta_{+}$in the introduction, and since $c^{\prime \prime}>c$, there exists $\delta>0$ such that $\beta_{+}\left(y, c^{\prime \prime}\right)-\beta_{+}(y, c)>\delta$ for all $y \in \bar{\omega}$. There exists now $N_{2}$ such that $\left|\beta_{1}(y, c)-\beta\left(x_{1}, y, c\right)\right| \leq \delta$ if $x_{1} \geq N_{2}, \forall y \in \bar{\omega}$. Hence, $B^{\prime} \geq 0$ for $x_{1} \geq N_{2}$ and $y \in \bar{\omega}$. Let us now define $N=\max \left(N_{1}, N_{2}\right)$ and translate $w_{+}$of $N$ to the right (we rename $w_{+}$this translated). The function $w_{+}$is now defined in $\left[N,+\infty\left[\times \bar{\omega}\right.\right.$ and satisfies $w_{+}(N, \cdot)=0$. Hence,

$$
\Delta w_{+}-\beta\left(x_{1}, y, c\right) \partial_{1} w_{+}+\int\left(x_{1}, w_{+}\right) \geq 0 \text { in }[N,+\infty[\times \omega
$$

For any $a>N$, we will show that the functions $u_{a}$ constructed in the step 1 are $\geq w_{+}$in $[N, a] \times \bar{\omega}$.

Otherwise, since $0<u_{a}<1$ in $(-a, a) \times \bar{\omega}$ and $w_{+}<1$, there exists a real $0 \leq \tau<a-N$ such that $u_{a}\left(x_{1}, y\right) \geq w_{+}\left(x_{1}-\tau, y\right)$ in $[N+\tau, a] \times \bar{\omega}$ with equality somewhere in $[N+\tau, a] \times \bar{\omega}$. Let us now define $z=u_{a}-w_{+}\left(x_{1}-\tau, y\right)$ in $\tilde{\Sigma}=[N+\tau, a] \times \bar{\omega}$. Thus, in $\tilde{\Sigma}$, the function $z$ satisfies

$$
\begin{aligned}
\Delta z- & \beta\left(x_{1}, y, c\right) \partial_{1} z+f\left(x_{1}, u_{a}\right)-f\left(x_{1}, w_{+}\left(x_{1}-\tau, y\right)\right) \\
= & -\Delta w_{+}\left(x_{1}-\tau, y\right)+\beta\left(x_{1}, y, c\right) \partial_{1} w_{+}\left(x_{1}-\tau, y\right) \\
& -f\left(x_{1}, w_{+}\left(x_{1}-\tau, y\right)\right) \\
\leq & -\Delta w_{+}\left(x_{1}-\tau, y\right)+\beta\left(x_{1}-\tau, y, c\right) \partial_{1} w_{+}\left(x_{1}-\tau, y\right) \\
& -f\left(x_{1}-\tau, w_{+}\left(x_{1}-\tau, y\right)\right)
\end{aligned}
$$

since $\beta$ is non increasing in $x_{1}, \partial_{1} w_{+} \geq 0$ and $f$ is non decreasing in $x_{1}$. The last expression is $\leq 0$ from (2.2) (since $x_{1}-\tau \geq N$ ). Hence, as $f$ is lipschitz-continuous in $u$, there exists a bounded function $c$ such that

$$
\left\{\begin{array}{c}
\Delta z-\beta\left(x_{1}, y, c\right) \partial_{1} z+c(x) z \leq 0 \text { in } \tilde{\Sigma} \\
\partial_{\nu} z=0 \text { on }(N+\tau, a) \times \partial \omega \\
z \geq 0 \text { in } \tilde{\Sigma} \text { with equality somewhere in }(N+\tau, a) \times \bar{\omega}
\end{array}\right.
$$

From the maximum principle and the Hopf lemma, we infer that $z \equiv 0$ in $\tilde{\Sigma}$ which is impossible for instance on $\{a\} \times \bar{\omega}$ since $w_{+}<1$.

Hence, $u_{a} \geq w_{+}$in $[N, a] \times \bar{\omega}$. By passage to the limit $a \rightarrow+\infty$, we conclude that $u \geq w_{+}$in $[N,+\infty[\times \bar{\omega}$, and lastly $u(+\infty, \cdot)=1$. 
Similarly, one can prove that $u \leq w_{-}$(for a translated of the function $\left.w_{-}\right)$in $\left.]-\infty,-N^{\prime}\right] \times \bar{\omega}$, for $N^{\prime}$ large enough. Thus, $u(-\infty, \cdot)=0$, this achieves the proof of theorem 1 in case II.

In case $I$, the arguments are the same up to technical details due to the different definition of the functions $f_{ \pm}^{\epsilon}$. Indeed, with the same notations, we have

$$
\begin{aligned}
& \Delta w_{+}-\beta\left(x_{1}, y, c\right) \partial_{1} w_{+}+f\left(x_{1}, w_{+}\right) \\
& =f\left(x_{1}, w_{+}\right)-f_{+}\left(w_{+}\right)+\epsilon \chi_{+}\left(w_{+}\right)\left(1-w_{+}\right) \\
& \quad+\left(\beta_{+}\left(y, c^{\prime}\right)-\beta\left(x_{1}, y, c\right)\right) \partial_{1} w_{+}
\end{aligned}
$$

It only remains tom show that the term $A=-f_{+}\left(w_{+}\right)+f\left(x_{1}, w_{+}\right)+$ $\epsilon \chi_{+}\left(w_{+}\right)\left(1-w_{+}\right)$is $\geq 0$ for $x_{1}$ large enough. If $w_{+} \leq \theta_{+}$, then $A=0$. If $\theta_{+} \leq w_{+} \leq\left(1+\theta_{+}\right) / 2$, we write

$$
A=\int_{\theta_{+}}^{w_{+}}\left(f_{u}^{\prime}\left(x_{1}, u\right)-f_{+}^{\prime}(u)\right) d u+\epsilon \chi_{+}\left(w_{+}\right)\left(1-w_{+}\right)
$$

For $x_{1} \geq N_{1}$ large enough, we have $\left|f_{u}^{\prime}\left(x_{1}, u\right)-f_{+}^{\prime}(u)\right| \leq \epsilon \delta_{+}\left(1-\theta_{+}\right) / 2$ (see the definition of $\delta_{+}$in the beginning of the step 2). Besides, $\chi_{+}\left(w_{+}\right)=\chi_{+}\left(w_{+}\right)-\chi_{+}\left(\theta_{+}\right) \geq \delta_{+}\left(w_{+}-\theta_{+}\right)$. Hence,

$$
A \geq-\epsilon\left(w_{+}-\theta_{+}\right) \delta_{+}\left(1-\theta_{+}\right) / 2+\epsilon \delta_{+}\left(w_{+}-\theta_{+}\right)\left(1-\theta_{+}\right) / 2 \geq 0
$$

If $\left(1+\theta_{+}\right) / 2 \leq w_{+} \leq 1$, we write $A=\int_{1}^{w_{+}}\left(f_{u}^{\prime}\left(x_{1}, u\right)-f_{+}^{\prime}(u)\right) d u+$ $\epsilon \chi_{+}\left(w_{+}\right)\left(1-w_{+}\right)$and conclude in the same way since $\chi_{+}\left(w_{+}\right) \geq$ $\min _{\left[\left(1+\theta_{+}\right) / 2,1\right]} \chi_{+}(u)>0$.

Hence, $u_{a}$ is greater than some fixed translated of $w_{+}$near $+\infty$. This yields that $u(+\infty, \cdot)=1$. Similarly, $u(-\infty, \cdot)=0$. This achieves the proof of theorem 1 in case $\mathrm{I}$.

\subsubsection{Case $c_{-}=c_{+}$, existence result}

From the results of $\S 2.1 .1$, this yiclds that $f\left(x_{1}, u\right)=f_{-}(u)=$ $f_{+}(u) \forall x_{1} \in \mathbb{R}, \forall u \in[0,1]$. Let us note $c=c_{-}=c_{+}$and $f(u)=f\left(x_{1}, u\right)$. The functions $u_{ \pm}$are solutions of

$$
\left\{\begin{array}{c}
\Delta u_{ \pm}-\beta_{ \pm}(y, c) \partial_{1} u_{ \pm}+f(u)=0 \text { in } \Sigma \\
\partial_{\nu} u_{ \pm}=0 \text { on } \partial \Sigma \\
u_{ \pm}(-\infty, \cdot)=0, u_{ \pm}(+\infty, \cdot)=1
\end{array}\right.
$$

Vol. 14, $n^{\circ} 5-1997$. 
Moreover, let us show that $u_{+}$and $u_{-}$are respectively super and subsolutions for problem $(P)$. Indeed

$\Delta u_{+}-\beta\left(x_{1}, y, c\right) \partial_{1} u_{+}+f\left(u_{+}\right)=\left(\beta_{+}(y, c)-\beta\left(x_{1}, y, c\right)\right) \partial_{1} u_{+} \leq 0$ in $\Sigma$

since $\beta$ is non increasing in $x_{1}$ and $\partial_{1} u_{+} \geq 0$. In the same way, we have

$$
\Delta u_{-}-\beta\left(x_{1}, y, c\right) \partial_{1} u_{-}+f\left(u_{-}\right) \geq 0 \text { in } \Sigma
$$

In both cases I and II, for the existence of a solution $u$ of $(P)$ such that $\partial_{1} u \geq 0$, it remains to prove that $u_{-} \leq u_{+}$in $\Sigma$ (up to translation) and to apply the results of [8] on the theory of sub- and supersolutions.

The behaviours of $u_{ \pm}$near $-\infty$ can be written as

$$
u_{ \pm}\left(x_{1}, y\right)=e^{\lambda_{ \pm} x_{1}} \phi_{ \pm}(y)+o\left(e^{\lambda_{ \pm} x_{1}}\right) \text { as } x_{1} \rightarrow-\infty
$$

where $\lambda_{ \pm}>0, \phi_{ \pm}>0$ on $\bar{\omega}$ are solutions of

$$
\Delta \phi_{ \pm}+\left(\lambda_{ \pm}^{2}-\lambda_{ \pm} \beta_{ \pm}(y, c)+f^{\prime}(0)\right) \phi_{ \pm}=0
$$

From the results of [7] used in $\S 2.1 .1$, since $\beta_{+}(\cdot, c) \leq \beta_{-}(\cdot, c)$, we have $0<\lambda_{+} \leq \lambda_{-}$.

If $\beta_{+}(\cdot, c) \equiv \beta_{-}(\cdot, c)$, then $\beta\left(x_{1}, y, c\right) \equiv \beta_{+}(y, c) \equiv \beta_{-}(y, c)$. From theorem 0 , the functions $u_{ \pm}$are equal up to translation, and all their translated are solutions of $(P)$.

Otherwise, $0<\lambda_{+}<\lambda$ and $u_{-} \ll u_{+}$as $x_{1} \rightarrow-\infty$. In the same way, we could prove that the exponents $\mu_{ \pm}$of the exponential behaviours of $1-u_{ \pm}$near $+\infty$ are such that $\mu_{+}<\mu_{-}<0$. Hence, $u_{-} \leq u_{+}$as $x_{1} \rightarrow \pm \infty$ and in $\bar{\Sigma}$ after translation; this achieves the proof of the existence of a solution $u$ of $(P)$ from the remarks above.

\subsection{Case III ("KPP" case)}

\subsubsection{Proof of the inequality $c_{-}^{*} \leq c_{+}^{*}$}

To prove this inequality, it only suffices to recall some results given in [7]. For a non-linearity $f$ of "KPP" type $C$, the minimal speed $c^{*}$ is namely obtained as the increasing limit as $\theta \searrow 0$ of the unique speeds $c_{\theta}$ corresponding to the function $f_{\theta}$ of "ignition temperature" type A defined by $f_{\theta}=f \chi_{\theta}$ where $\chi_{\theta}$ is a smooth and non negative function such that $\chi_{\theta} \equiv 0$ on $[0, \theta]$ and $\chi_{\theta} \equiv 1$ on $[2 \theta, 1]$.

In the situation of case III, since $0 \leq f_{-} \leq f_{+} \leq 1$, it comes that $f_{-}^{\theta}=f_{-} \chi_{\theta} \leq f_{+}^{\theta}=f_{+} \chi_{\theta}$. Hence, from $\S 2.1 .1$, the unique speeds $c_{ \pm}^{\theta}$ 
corresponding to the functions $f_{ \pm}^{\theta}$ are such that $c_{-}^{\theta} \leq c_{+}^{\theta}$. By passage to the limit $\theta \searrow 0$, we conclude that $c_{-}^{*} \leq c_{+}^{*}$.

\subsubsection{Existence of solutions for any $c \geq c_{+}^{*}$}

In case III, we have $f_{ \pm}>0$ on $(0,1)$. For each non-linearity $f_{-}$and $f_{+}$, there exist minimal speeds $c_{-}^{*}$ and $c_{+}^{*}$ such that $c_{-}^{*} \leq c_{+}^{*}$ (from 32.2 .1 .).

Let $h$ be fixed in $(0,1)$ and $c \geq c_{+}^{*}$. Let $a>0$ and $R_{a}=(-a, a) \times \omega$ the finite cylinder. By a method of sub- and super-solutions, we will first show the existence of solutions $\left(u_{a}\right)$ of the problems

$$
\left\{\begin{array}{c}
\Delta u_{a}-\beta\left(x_{1}, y, c\right) \partial_{1} u_{a}+f\left(x_{1}, u_{a}\right)=0 \text { in } R_{a} \\
\partial_{\nu} u_{a}=0 \text { on }(-a, a) \times \partial \omega \\
\max _{\bar{\omega}} u_{a}(0, \cdot)=h
\end{array}\right.
$$

Indeed, we know that there exists $u_{+}^{*}$ such that $\partial_{1} u_{+}^{*}>0$ in $\bar{\Sigma}$ solution of

$$
\left\{\begin{array}{c}
\Delta u_{+}^{*}-\beta_{+}\left(y, c_{+}^{*}\right) \partial_{1} u_{+}^{*}+f_{+}\left(u_{+}^{*}\right)=0 \text { in } \Sigma \\
\partial_{\nu} u_{+}^{*}=0 \text { on } \partial \Sigma \\
u_{+}^{*}(-\infty, \cdot)=0, u_{+}^{*}(+\infty, \cdot)=1
\end{array}\right.
$$

Since $c \geq c_{+}^{*}$ and $\beta$ is non increasing in $x_{1}$, we have $\beta_{+}\left(y, c_{+}^{*}\right)<$ $\beta_{+}(y, c) \leq \beta\left(x_{1}, y, c\right)$. Moreover, $\partial_{1} u_{+}^{*}>0$, and $f\left(x_{1}, u_{+}^{*}\right) \leq f_{+}\left(u_{+}^{*}\right)$ because $f$ is non decreasing in $x_{1}$. Hence, it comes that

$$
\left\{\begin{array}{c}
\Delta u_{+}^{*}-\beta\left(x_{1}, y, c\right) \partial_{1} u_{+}^{*}+f\left(x_{1}, u_{+}^{*}\right) \leq 0 \text { in } R_{a} \\
\partial_{\nu} u_{+}^{*}=0 \text { on }(-a, a) \times \partial \omega
\end{array}\right.
$$

Hence, $u_{+}^{*}$ is a super-solution of problem $\left(P_{a}\right)$ (without the normalization condition on $\{0\} \times \bar{\omega})$ such that $\partial_{1} u_{+}^{*}>0$. Besides, each constant $h_{a}=\min _{\{-a\} \times \bar{\omega}} u_{+}^{*}$ is a subsolution of this problem because $f\left(x_{1}, h_{a}\right) \geq$ $f_{-}\left(h_{a}\right) \geq 0$. Since $\beta$ is non increasing in $x_{1}$ and $f$ is non decreasing in $x_{1}$, all the hypotheses required for the application of general theorem 7.2 of [8] are satisfied. There exists thus a unique solution $v_{a}$ of the problem

$$
\left\{\begin{array}{c}
\Delta v_{a}-\beta\left(x_{1}, y, c\right) \partial_{1} v_{a}+f\left(x_{1}, v_{a}\right)=0 \text { in } R_{a} \\
\partial_{\nu} v_{a}=0 \text { on }(-a, a) \times \partial \omega \\
v_{a}(-a, \cdot)=h_{a}, v_{a}(+a, \cdot)=u_{+}^{*}(+a, \cdot)
\end{array}\right.
$$

with $\partial_{1} v_{a}>0$ in $(-a, a) \times \bar{\omega}$.

Of course, we can do this work for any translated function of $u_{+}^{*}$, i.e. for the functions $u_{+, \tau}^{*}:\left(x_{1}, y\right) \mapsto u_{+}^{*}\left(x_{1}+\tau, y\right)$ for any $\tau \in \mathbb{R}$. We would obtain functions $v_{a, \tau}$ by the same way. From the uniqueness results above, 
from the classical a priori elliptic estimates and by a compactness argument, the functions $v_{a, \tau}$ are continuous in $\tau$. Since $h_{a, \tau}-\min _{\{-a\} \times \bar{w}} u_{+, \tau}^{*} \rightarrow 1$ as $\tau \rightarrow+\infty$, and $\max u_{a}(a, \cdot) \rightarrow 0$ as $\tau \rightarrow-\infty$, it comes that there exists $\tau \in \mathbb{R}$ such that $u_{a}^{\bar{\omega}}:=v_{a, \tau}$ is solution of the initial problem $\left(P_{a}\right)$, with the normalization condition $\max _{\bar{\omega}} u_{a}(0)=$.$h .$

As usual, one passes to the limit $a \rightarrow+\infty$. For some subsequence, one has $u_{a} \rightarrow u$ in $C_{l o c}^{1, \mu}(\bar{\Sigma})$ such that

$$
\left\{\begin{array}{c}
\Delta u-\beta\left(x_{1}, y, c\right) \partial_{1} u+f\left(x_{1}, u\right)=0 \text { in } \Sigma \\
\partial_{\nu} u=0 \text { on } \partial \Sigma \\
\max _{\bar{\omega}} u(0, \cdot)=h
\end{array}\right.
$$

and $\partial_{1} u \geq 0$ in $\bar{\Sigma}$. There exist functions $\psi_{ \pm}$defined on $\bar{\omega}$ such that $u( \pm \infty, y)=\psi_{ \pm}(y) \forall y \in \bar{\omega}$, and $\psi_{ \pm}$satisfy the limit problems

$$
\left\{\begin{array}{c}
\Delta \psi_{ \pm}+f_{ \pm}\left(\psi_{ \pm}\right)=0 \text { in } \omega \\
\partial_{\nu} \psi_{ \pm}=0 \text { on } \partial \omega
\end{array}\right.
$$

By integration of these equations and since $u$ is increasing in $x_{1}$, it comes immediately that $\psi_{-} \equiv 0$ and $\psi_{+} \equiv 1$.

Remark. - The methods developped in $\$ 2.1 .2$. in cases I and II do not work in case III. One could answer whether there exists a solution of $(P)$ for any $c \geq c_{-}^{*}$ or even only for $c>c_{--}^{*}$. This question is still open.

\section{NECESSARY CONDITIONS, PROOFS OF THEOREMS 2, 3, 4}

\subsection{Bounds on the eventual speeds $c$ solutions: proof of theorem 2 \\ 3.1.1. Cases I and II \\ ("ignition temperature" or "bistable" cases), proof of inequality $c_{-} \leq c \leq c_{-}$}

Let us suppose that there exists a solution $(c, u)$ of $(P)$ such that $\partial_{1} u \geq 0$ and $c>c_{+}$. Since $f\left(x_{1}, u\right) \leq f_{+}(u), \beta\left(x_{1}, y, c\right) \geq \beta_{+}(y, c)$ and $\partial_{1} u \geq 0$, it comes that

$$
\Delta u-\beta_{+}(y, c) \partial_{1} u+f_{+}(u) \geq 0 \text { in } \Sigma
$$

Let us remark that

$$
\Delta u_{+}-\beta_{+}(y, c) \partial_{1} u_{+}+f_{+}\left(u_{+}\right) \leq 0
$$


because we have supposed that $c>c_{+}$, and since $\partial_{1} u_{+} \geq 0$. In order to obtain a contradiction, we will firstly compare $u$ and $u_{+}$in $\pm \infty$ and secondly we will use a sliding method. This approach is general and is available in both cases I and II. Other proofs are given in [11] and [17].

Study of $u$ and $u_{+}$near $-\infty$

Case I: the non-linearity $f_{+}$is of type A and $f_{+} \equiv 0$ on $\left[0, \theta_{+}\right]$. Berestycki and Nirenberg proved in [7] that the behaviour of the function $u_{+}$solution of $\left(P_{+}\right)$is given by

$$
u_{+}\left(x_{1}, y\right)=e^{\lambda_{+} x_{1}} \phi_{+}(y)+o\left(e^{\lambda_{+} x_{1}}\right) \text { as } x_{1} \rightarrow-\infty
$$

where the real $\lambda_{+}$is $>0$, the function $\phi_{+}>0$ on $\bar{\omega}$. The function $w_{+}=e^{\lambda_{+} x_{1}} \phi_{+}(y)$ is solution of

$$
\left\{\begin{array}{c}
\Delta w_{+}-\beta_{+}\left(y, c_{+}\right) \partial_{1} w_{+}=0 \text { in } \Sigma \\
\partial_{\nu} w_{+}=0 \text { on } \partial \Sigma
\end{array}\right.
$$

From (3.1) and the profile of $f_{+}$, we have

$$
\left.\left.\Delta u-\beta_{+}(y, c) \partial_{1} u \geq 0 \text { in }\right]-\infty,-N\right] \times \omega
$$

for $N$ large enough. On the other side, as $c>c_{+}$, we have $\int_{\omega} \beta_{+}(y, c) d y>$ $\int_{\omega} \beta_{+}\left(y, c_{+}\right) d y>0$ (by integration of the equation satisfied by $\left.u_{+}\right)$. Hence, from the results of [7], there exists a function $w$ of the form $w=e^{\lambda x_{1}} \phi(y)$ with $\lambda>\lambda_{+}>0$ and $\phi>0$ on $\bar{\omega}$ solution of

$$
\left\{\begin{array}{c}
\Delta w-\beta_{+}(y, c) \partial_{1} w=0 \text { in } \Sigma \\
\partial_{\nu} w=0 \text { on } \partial \Sigma
\end{array}\right.
$$

Thus, the function $z=u-w$ satisfies

$$
\left\{\begin{array}{c}
\left.\left.\Delta z-\beta_{+}(y, c) \partial_{1} z \geq 0 \text { in }\right]-\infty,-N\right] \times \omega \\
\partial_{\nu} z=0 \text { on }(-\infty,-N) \times \partial \omega
\end{array}\right.
$$

and after multiplication of $w$ by a positive constant large enough, one can suppose that $u \leq w$ on $\{-N\} \times \bar{w}$. The maximum principle and Hopf lemma yield

$$
u \leq w \text { in }]-\infty,-N] \times \bar{\omega}
$$

On the other side, we have recalled that $u_{+} \sim e^{\lambda_{+} x_{1}} \phi_{+}(y)$ as $x_{1} \rightarrow-\infty$, with $\phi_{+}>0$ on $\bar{\omega}$ and $\lambda>\lambda_{+}>0$. Thus, it results from (3.2) and (3.3) that for $N^{\prime}$ large enough, we have

$$
\left.\left.u<u_{+} \text {in }\right]-\infty,-N^{\prime}\right] \times \bar{\omega}
$$

Vol. 14, n $5-1997$ 
Case II: at first, the function $u_{+}$solution of $\left(P_{+}\right)$has the same behaviour in $-\infty$ as in case I.

The source term $f_{+}$is now of type $B$, hence $f_{+}^{\prime}(0)<0$, and from the results of [7], for all $0 \leq \epsilon<-f_{+}^{\prime}(0)$, there exists a function $w_{\epsilon}=e^{\lambda_{\epsilon} x_{1}} \phi_{\epsilon}(y)$ with $\lambda_{\epsilon}>0$ and $\phi_{\epsilon}>0$ on $\bar{\omega}$ solution of

$$
\left\{\begin{array}{c}
\Delta w_{\epsilon}-\beta_{+}(y, c) \partial_{1} w_{\epsilon}+\left(f_{+}^{\prime}(0)+\epsilon\right) w_{\epsilon}=0 \text { in } \Sigma \\
\partial_{\nu} w_{\epsilon}=0 \text { on } \partial \Sigma
\end{array}\right.
$$

The real $\lambda_{\epsilon}$ is the unique positive solution of the equation

$$
\lambda_{\epsilon}^{2}=\mu_{1}\left(-\Delta+\lambda_{c} \beta_{\cdot}(y, c)-f_{+}^{\prime}(0)-\epsilon\right)
$$

where in a general way, $\mu_{1}(-L)$ designates the principal eigenvalue of the elliptic operator $L$ with Neumann boundary conditions. Thus, $\lambda_{\epsilon}$ is continuous in $\epsilon$ and for $\epsilon=0$, we have $\lambda_{0}=\lambda>0$ such that $\lambda^{2}=\mu_{1}\left(-\Delta+\lambda \beta_{+}(y, c)-f_{+}^{\prime}(0)\right)$.

On the other hand, since $c>c_{+}$(which implies $\beta_{+}(y, c)<\beta_{+}\left(y, c_{+}\right)$), we have already remarked that $\lambda>\lambda_{+}$(like in case I). Hence, for $0<\epsilon_{0}<-f_{+}^{\prime}(0)$ small enough, we infer $\lambda_{\epsilon_{0}}>\lambda_{+}$.

As $u \underset{x_{1} \rightarrow-\infty}{\longrightarrow} 0$ uniformly in $y \in \bar{\omega}$ and $\Delta u-\beta_{+}(y, c) \partial_{1} u+f_{+}(u) \geq 0$, there exists $N$ large enough such that

$$
\left.\left.\Delta u-\beta_{+}(y, c) \partial_{1} u+\left(f_{+}^{\prime}(0)+\epsilon_{0}\right) u \geq 0 \text { in }\right]-\infty,-N\right] \times \omega
$$

Thus,

$$
\left\{\begin{array}{c}
\Delta\left(u-w_{\epsilon_{0}}\right)-\beta_{+}(y, c) \partial_{1}\left(u-w_{\epsilon_{0}}\right)+\left(f_{+}^{\prime}(0)+\epsilon_{0}\right)\left(u-w_{\epsilon_{0}}\right) \geq 0 \\
\text { in }]-\infty, \quad N] \times \omega \\
\partial_{\nu}\left(u-w_{\epsilon_{0}}\right)=0 \text { on }(-\infty,-N) \times \partial \omega
\end{array}\right.
$$

Since $f_{+}^{\prime}(0)+\epsilon_{0}<0$, multiplying $w_{\epsilon_{0}}$ by a positive constant in order that $u \leq w_{\epsilon_{0}}$ on $\{-N\} \times \bar{\omega}$, it follows from the maximum principle and Hopf lemma that $u \leq w_{\epsilon_{0}}=e^{\lambda_{\epsilon_{0}} x_{1}} \phi_{\epsilon_{0}}(y)$ in $\left.]-\infty,-N\right] \times \bar{\omega}$. But, $\lambda_{\epsilon_{0}}>\lambda_{+}$and $u_{+} \sim_{x_{1} \rightarrow-\infty} e^{\lambda_{+} x_{1}} \phi_{+}(y)$. Hence, as in the first case, one concludes that

$$
\left.\left.u<u_{+} \text {in }\right]-\infty,-N^{\prime}\right] \times \bar{\omega} \text { for } N^{\prime} \text { large enough }
$$

Study of $u$ and $u_{+}$near $+\infty$

In both cases I and II, from the results of [7], the asymptotic behaviour of the function $u_{+}$solution of problem $\left(P_{+}\right)$as $x_{1} \rightarrow+\infty$ is given by

$$
u_{+}=1-e^{\mu_{+} x_{1}} \psi_{+}(y)+o\left(e^{\mu_{+} x_{1}}\right) \text { as } x_{1} \rightarrow+\infty
$$


where $\mu_{+}<0, \psi_{+}>0$ on $\bar{\omega}$ and $W_{+}=e^{\mu_{+} x_{1}} \psi_{+}(y)$ are solutions of

$$
\left\{\begin{array}{c}
\Delta W_{+}-\beta_{+}\left(y, c_{+}\right) \partial_{1} W_{+}+f_{+}^{\prime}(1) W_{+}=0 \text { in } \Sigma \\
\partial_{\nu} W_{+}=0 \text { on } \partial \Sigma
\end{array}\right.
$$

In both cases I and II, we systematically have $f_{+}^{\prime}(1)<0$. As above, for any $\epsilon>0$, there exists a function $w_{\epsilon}^{\prime}=e^{\mu_{c} x_{1}} \psi_{\epsilon}(y)$, where the real $\mu_{\epsilon}$ is $<0$ and the function $\psi_{\epsilon}>0$ on $\bar{\omega}$, solution of

$$
\left\{\begin{array}{c}
\Delta w_{\epsilon}^{\prime}-\beta_{+}(y, c) \partial_{1} w_{\epsilon}^{\prime}+\left(f_{+}^{\prime}(1)-\epsilon\right) w_{\epsilon}^{\prime}=0 \text { in } \Sigma \\
\partial_{\nu} w_{\epsilon}^{\prime}=0 \text { on } \partial \Sigma
\end{array}\right.
$$

where the real $\mu_{\epsilon}$ is the unique negative solution of

$$
\mu_{c}^{2}=\mu_{1}\left(-\Delta+\mu_{\epsilon} \beta_{+}(y, c)-\left(f_{1}^{\prime}(1)-\epsilon\right)\right)
$$

The eigenvalue $\mu_{\epsilon}$ depends thus continuously on $\epsilon>0$. But, for $\epsilon=0$ we have $\mu_{0}=\mu$ such that $\mu^{2}=\mu_{1}\left(-\Delta+\mu \beta_{+}(y, c)-f_{+}^{\prime}(1)\right)$. Since $c>c_{+}$ (which yields $\beta_{+}(y, c)>\beta_{+}\left(y, c_{+}\right)$), if follows from theorem $2.1 \mathrm{e}$ ) of [7] that $\mu_{+}<\mu<0$ and then for $\epsilon_{1}>0$ close enough to 0 , we have

$$
\mu_{+}<\mu_{\epsilon_{1}}<0
$$

On the other side, from (3.1), the function $u$ satisfies

$$
\Delta(1-u)-\beta_{+}(y, c) \partial_{1}(1-u)-f_{+}(u) \leq 0 \text { in } \Sigma
$$

and $\left(f_{+}^{\prime}(1)-\epsilon_{1}\right)(1-u) \leq-f_{+}(u)$ in the neighbourhood of $u=1$ that is to say if $x_{1}$ is large enough. Hence, for $N$ large enough, we have

$$
\left\{\begin{array}{c}
\Delta(1-u)-\beta_{+}(y, c) \partial_{1}(1-u)+\left(f_{+}^{\prime}(1)-\epsilon_{1}\right)(1-u) \leq 0 \text { in }[N,+\infty[\times \omega \\
\partial_{\nu}(1-u)=0 \text { on } \partial \Sigma
\end{array}\right.
$$

This implies that the function $1-u-w_{\epsilon_{1}}^{\prime}$ satisfies

$$
\left\{\begin{array}{c}
\Delta\left(1-u-w_{\epsilon_{1}}^{\prime}\right)-\beta_{+}(y, c) \partial_{1}\left(1-u-w_{\epsilon_{1}}^{\prime}\right)+\left(f_{+}^{\prime}(1)-\epsilon_{1}\right)\left(1-u-w_{\epsilon_{1}}^{\prime}\right) \\
\leq 0 \text { in }[N,+\infty \mid \times \omega \\
\partial_{\nu}\left(1-u-w_{\epsilon_{1}}^{\prime}\right)=0 \text { on } \partial \Sigma
\end{array}\right.
$$

From the maximum principle and Hopf lemma, since $f_{+}^{\prime}(1)-\epsilon_{1}<0$, we can conclude as above that

$$
1-u>w_{\epsilon_{1}}^{\prime}=e^{\mu_{\epsilon_{1}} x_{1}} \psi_{\epsilon_{1}}(y) \gg e^{\mu_{+} x_{1}} \psi_{+}(y) \sim 1-u_{+} \text {near }+\infty
$$

It follows that $u<u_{+}$in the neighbourhood of $+\infty$. 
As a consequence of the arguments above, in each case I and II, there exists $R>0$ such that

$$
\left.u<u_{+} \text {on }(]-\infty,-R\right] \cup[R,+\infty[) \times \bar{\omega}
$$

Since $u_{+}$is increasing in $x_{1}$ and $u$ is bounded by a constant $\beta<1$ on the compact set $[-R,+R] \times \bar{\omega}$, there exists $\tau>0$ such that

$$
u<u_{+}\left(x_{1}+\tau, y\right) \quad \forall\left(x_{1}, y\right) \in \bar{\Sigma}
$$

As the behaviours of $u$ and $u_{+}$are exponentially different in $\pm \infty$, one can translate the graph of $u_{+}(\cdot+\tau, \cdot)$ to the right in such a way that

$$
u \leq u_{+}\left(x_{1}+s, y\right) \text { in } \bar{\Sigma}
$$

with equality somewhere in $\bar{\Sigma}$. Thus, the function $z=u-u_{+}(\cdot+s, \cdot)$ satisfies

$$
\left\{\begin{array}{c}
\Delta z-\beta\left(x_{1}, y, c\right) \partial_{1} z+f\left(x_{1}, u\right)-f_{+}\left(u_{+}\left(x_{1}+s, y\right)\right) \\
=\left(\beta\left(x_{1}, y, c\right)-\beta_{+}\left(y, c_{+}\right)\right) \partial_{1} u_{+}\left(x_{1}+s, y\right) \text { in } \Sigma \\
\partial_{\nu} z=0 \text { on } \partial \Sigma
\end{array}\right.
$$

Since $\beta\left(x_{1}, y, c\right) \geq \beta_{+}(y, c) \geq \beta_{+}\left(y, c_{+}\right) \quad(\beta$ is non increasing in $x_{1}$ and $\left.c>c_{+}\right)$and since $\partial_{1} u_{+} \geq 0, f_{+}(u) \geq f\left(x_{1}, u\right)$ and $f_{+}$ is lipschitz-continuous, there exists a bounded function $c$ such that $f_{+}(u)-f_{+}\left(u_{+}\left(x_{1}+s, y\right)\right)=c(x) z$, and

$$
\Delta z-\beta\left(x_{1}, y, c\right) \partial_{1} z+c(x) z \geq 0 \text { in } \bar{\Sigma}
$$

Since $z \leq 0$ with equality somewhere in $\bar{\Sigma}$, it results from the maximum principle and Hopf lemma that $z \equiv 0$. That is a contradiction with the behaviours of $u$ and $u_{+}$as $x_{1} \rightarrow \pm \infty$.

Remark. - We would obtain a similar contradiction if we supposed that $c<c_{-}$.

\subsubsection{Case III: proof of inequality $c \geq c_{-}^{*}$}

'The proof is identical to this of $[7](\S 8)$. One supposes the contrary, that is to say $c<c_{-}^{*}$. Thus, with the notations of $\$ 2.2 .1$., for $\epsilon<0$ and $\theta>0$ close to 0 , we have $c<c_{\theta}^{\epsilon}$ where $\left(c_{\theta}^{\epsilon}, u_{\theta}^{\epsilon}\right)$ is the unique pair solution of

$$
\left\{\begin{array}{c}
\Delta u_{\theta}^{\epsilon}-\beta_{-}\left(y, c_{\theta}^{\epsilon}\right) \partial_{1} u_{\theta}^{\epsilon}+\left(f_{-} \chi_{\theta}\right)\left(u_{\theta}^{\epsilon}\right)=0 \text { in } \Sigma \\
\partial_{\nu} u_{\theta}^{\epsilon}=0 \text { on } \partial \Sigma \\
u_{\theta}^{\epsilon}(-\infty, \cdot)=-\epsilon<u_{\theta}^{\epsilon}<u_{\theta}^{\epsilon}(+\infty, \cdot)=1
\end{array}\right.
$$


where $\chi_{\theta}$ is a smooth function, $\geq 0$, such that $\chi_{\theta} \equiv 0$ on $[0, \theta]$ and $\chi_{\theta} \equiv 1$ on $[2 \theta, 1]$. The function $f_{-}$is extended on $\mathbb{R}_{-}$by $f_{-} \equiv 0$ on $\mathbb{R}_{-}$.

As in the proofs of $\S 3.1 .1$, up to translation, one has $u_{\theta}^{\epsilon} \leq u$ with equality somewhere in $\bar{\Sigma}$. But,

$$
\left\{\begin{array}{c}
\Delta\left(u_{\theta}^{\epsilon}-u\right)-\beta\left(x_{1}, y, c\right) \partial_{1}\left(u_{\theta}^{\epsilon}-u\right)+\left(\chi_{\theta} f_{-}\right)\left(u_{\theta}^{\epsilon}\right)-\left(\chi_{\theta} f_{-}\right)(u) \\
=\left(\beta_{-}\left(y, c_{\theta}^{\epsilon}\right)-\beta\left(x_{1}, y, c\right)\right) \partial_{1} u_{\theta}^{\epsilon} \\
\geq 0 \quad \geq 0 \\
+\left(f\left(x_{1}, u\right)-\chi_{\theta} f_{-}(u)\right) \geq 0 \text { in } \Sigma \\
\geq 0 \quad \partial_{\nu}\left(u_{\theta}^{\epsilon}-u\right)=0 \text { on } \partial \Sigma
\end{array}\right.
$$

Thus, $u_{\theta}^{\epsilon} \equiv u$. This is impossible from the behaviours of these functions as $x_{1} \rightarrow-\infty$.

\subsection{The solutions are increasing in $x_{1}$ : proof of theorem 3}

With the assumptions on the behaviours of the functions $\beta$ and $f$ near $\pm \infty$, and $f$ as $u \rightarrow 0$ and 1 , assumptions which we assume in all what follows, some of the properties of monotonicity and partial uniqueness enounced in theorem 0 for problem $\left(P_{i n v}\right)$ are preserved in the non invariant by translation case. We will use the results of the appendix on the asymptotic behaviour of solutions, which are a little stronger than what we actually need in this section.

Let $(c, u)$ a solution of $(P)$. From the maximum principle and the Hopf lemma, it immediately comes that $0<u<1$ in $\bar{\Sigma}$. All the hypotheses of theorems 1 and 2 of the appendix are required (behaviours of $\beta$ and $f$ as $x_{1} \rightarrow \pm \infty$ and $f_{-}$and $f_{+}$near 0 and 1 respectively). The principal eigenvalue of the elliptic operator $-\Delta_{y}-f_{-}^{\prime}(0)$ with Neumann boundary conditions is $\mu_{1}=-f_{--}^{\prime}(0)$ with principal eigenfunction $\sigma=1$. In cases II and III, we respectively have $\mu_{1}>0$ and $\mu_{1}<0$.

In case $1, \mu_{1}=0$ and $h\left(x_{1}, s\right):=f\left(x_{1}, s\right)-f_{-}^{\prime}(0) s \equiv 0$ for $0 \leq s \leq \theta_{+}$. In case I, in order to apply theorem 1 of the appendix for the behaviour of $u$ as $x_{1} \rightarrow-\infty$, it only remains to prove $\int \beta_{-}(y, c)>0(\sigma \equiv 1$ is the first eigenfunction of $-\Delta$ ). Let us integrate the equation satisfied by $u$ in $\bar{\Sigma}$. Since $\partial_{1} u \rightarrow 0$ as $\left|x_{1}\right| \rightarrow+\infty$ (from the classical a priori estimates) and since $\partial_{\nu} u=0$, the integral $\int_{\Sigma} \Delta u$ exists and $=0$. Hence,

$$
0<\int_{\Sigma} f\left(\begin{array}{c}
\left.x_{1}, y, u\right), \not \equiv 0 \\
\substack{\geq 0 \\
a \rightarrow+\infty}
\end{array} \int_{\omega} \int_{-a}^{a} \beta\left(x_{1}, y, c\right) \partial_{1} u\left(x_{1}, y\right)\right.
$$


By integration by parts, it comes

$$
\begin{aligned}
\int_{\omega} & \int_{-a}^{a} \beta\left(x_{1}, y, c\right) \partial_{1} u\left(x_{1}, y\right) d x_{1} d y \\
= & \int_{\omega}(\beta(a, y, c) u(a, y)-\beta(-a, y, c) u(-a, y)) d y \\
& +\int_{\omega} \int_{-a}^{a}-\beta_{x_{1}}\left(x_{1}, y, c\right) u\left(x_{1}, y\right) d x_{1} d y
\end{aligned}
$$

From the uniform convergences of $\beta$ to $\beta_{ \pm}$and $u$ to 0 and 1 as $x_{1} \rightarrow \pm \infty$, it comes that the first term converges to $\int_{\omega} \beta_{+}(y, c) d y$. In the second term, we have $\beta_{x_{1}}\left(x_{1}, y, c\right) \geq 0$, and $0 \leq u \leq 1$. Hence,

$$
\begin{aligned}
0 & \leq \int_{\omega} \int_{-a}^{a}-\beta_{x_{1}}\left(x_{1}, y, c\right) u\left(x_{1}, y\right) d x_{1} d y \\
& \leq \int_{\omega} \int_{-a}^{a}-\beta_{x_{1}}\left(x_{1}, y, c\right) d x_{1} d y \\
& =\int_{\omega}(\beta(-a, y, c)-\beta(a, y, c)) d y \rightarrow \int_{\omega}\left(\beta_{-}(y, c)-\beta_{+}(y, c)\right) d y
\end{aligned}
$$

Lastly, it follows that

$$
0<\int_{\Sigma} f\left(x_{1}, y, u\right) \leq \int_{\omega} \beta_{-}(y, c) d y
$$

Hence, from theorem 1 of the appendix applied in cases I, II or III, the behaviour of $u$ near $-\infty$ is

$$
\left\{\begin{aligned}
u\left(x_{1}, y\right) & =e^{\lambda x_{1}} \phi(y)+o\left(e^{\lambda x_{1}}\right) \\
\nabla u\left(x_{1}, y\right) & =\nabla\left(e^{\lambda x_{1}} \phi(y)\right)+o\left(e^{\lambda x_{1}}\right)
\end{aligned} \text { as } x_{1} \rightarrow-\infty\right.
$$

or may be in case III,

$$
\left\{\begin{aligned}
u\left(x_{1}, y\right) & =e^{\lambda x_{1}}\left(-x_{1} \phi(y)+\phi_{0}(y)\right)+o\left(e^{\lambda x_{1}}\right) \\
\nabla u\left(x_{1}, y\right) & =\nabla\left(e^{\lambda x_{1}}\left(-x_{1} \phi(y)+\phi_{0}(y)\right)\right)+o\left(e^{\lambda x_{1}}\right)
\end{aligned} \text { as } x_{1} \rightarrow-\infty\right.
$$

with anyway $\lambda>0, \phi>0$ on $\bar{\omega}$ solutions of

$$
\left\{\begin{array}{c}
\Delta \phi+\left(\lambda^{2}-\lambda \beta_{-}(y, c)+f_{-}^{\prime}(0)\right) \phi=0 \text { in } \omega \\
\partial_{\nu} \phi=0 \text { on } \partial \omega
\end{array}\right.
$$

In order to study the behaviour as $x_{1} \rightarrow+\infty$, we investigate the operator $-\Delta_{y}-f_{+}^{\prime}(1)$. Its principal eigenvalue is $-f_{+}^{\prime}(1)>0$ in each case I, II and III. From theorem 2 of the appendix, it comes that

$$
\left\{\begin{array}{c}
u\left(x_{1}, y\right)=1-e^{\mu \cdot x_{1}} \psi(y)+o\left(e^{\mu x_{1}}\right) \\
\nabla u\left(x_{1}, y\right)=-\nabla\left(e^{\mu x_{1}} \psi(y)\right)+o\left(e^{\mu \cdot x_{1}}\right)
\end{array} \text { as } x_{1} \rightarrow+\infty\right.
$$


with anyway $\mu<0, \psi>0$ on $\bar{\omega}$ solutions of

$$
\left\{\begin{array}{c}
\Delta \psi+\left(\mu^{2}-\mu \beta_{+}(y, c)+f_{+}^{\prime}(1)\right) \psi=0 \text { in } \omega \\
\partial_{\nu} \psi=0 \text { on } \partial \omega
\end{array}\right.
$$

We can thus see that for $R$ large enough, we have $\partial_{1} u>0$ if $\left|x_{1}\right| \geq R$, for all $y \in \bar{\omega}$. Since $0<u<1$ in $\bar{\Sigma}$ and from the growth of $u$ near $\pm \infty$, we can even say that there exists some $R_{0} \geq R$ such that

$$
u\left(-R_{0}, y\right)<u\left(x_{1}, y\right)<u\left(R_{0}, y\right) \quad \forall\left(x_{1}, y\right) \subset\left(R_{0}, R_{0}\right) \times \bar{\omega}
$$

As $\beta$ is decreasing in $x_{1}$ and $f$ is increasing in $x_{1}$, we can apply general theorem 2.1 of [8] on the sliding method and obtain directly that $u$ is increasing in $x_{1}$ in $\left(-R_{0}, R_{0}\right) \times \bar{\omega}$ and finally that $\partial_{1} u \geq 0$ in $\bar{\Sigma}$.

Moreover, since $f$ and $\beta$ are derivable and lipschitz-continuous with respect to $x_{1}$, one can derivate the equation satisfied by $u$. From the strong maximum principle and Hopf lemma, we infer that $\partial_{1} u>0$ in $\bar{\Sigma}$. That ends the proof of theorem 3.

\subsection{Structure of the solutions in cases I and II: proof of theorem 4}

\subsubsection{Comparison between solutions with different speeds}

Let us only consider in this section the cases I and II ("ignition temperature" or "bistable" cases). Let us suppose that $(c, u)$ and $\left(c^{\prime}, u^{\prime}\right)$ are solutions of $(P)$ with speeds $c<c^{\prime}$. As in $\S 3.2$, the behaviours of $u$ and $u^{\prime}$ as $x_{1} \rightarrow \pm \infty$ can be explicited

$$
\begin{gathered}
u\left(x_{1}, y\right)=e^{\lambda x_{1}} \phi(y)+o\left(e^{\lambda x_{1}}\right) \text { as } x_{1} \rightarrow-\infty \\
u^{\prime}\left(x_{1}, y\right)=e^{\lambda^{\prime} x_{1}} \phi^{\prime}(y)+o\left(e^{\lambda^{\prime} x_{1}}\right) \text { as } x_{1} \rightarrow-\infty
\end{gathered}
$$

with constants $\lambda, \lambda^{\prime}>0$ and functions $\phi, \phi^{\prime}>0$ on $\bar{\omega}$. The functions $w=e^{\lambda x_{1}} \phi(y)$ and $w^{\prime}=e^{\lambda^{\prime} x_{1}} \phi^{\prime}(y)$ are solutions of the linearized limit problems

$$
\left\{\begin{array}{c}
\Delta w-\beta_{-}(y, c) \partial_{1} w+f_{-}^{\prime}(0) w=0 \text { in } \Sigma \\
\Delta w^{\prime}-\beta_{-}\left(y, c^{\prime}\right) \partial_{1} w^{\prime}+f_{-}^{\prime}(0) w^{\prime}=0 \text { in } \Sigma \\
\partial_{\nu} w=\partial_{\nu} w^{\prime}=0 \text { on } \partial \Sigma
\end{array}\right.
$$

From theorem 2.1e) of [7], since $c<c^{\prime}$, we have $0<\lambda<\lambda^{\prime}$.

Hence, $u^{\prime}<u$ as $x_{1} \rightarrow-\infty$. Similarly, we could obtain the same comparaison as $x_{1} \rightarrow+\infty$.

We actually want to prove that $u^{\prime}<u$ in $\bar{\Sigma}$. If this does not occur, as the behaviours of $u$ and $u^{\prime}$ are exponentially different in $\pm \infty$, one can translate 
the function $u$ to the left such that its graph touches this of $u^{\prime}$. In other words, there exists a real $\tau \geq 0$ such that $u_{\tau}\left(x_{1}, y\right)=u\left(x_{1}+\tau, y\right) \geq u^{\prime}$ in $\bar{\Sigma}$ with equality somewhere. Hence, the function $z=u_{\tau}-u^{\prime}$ is $\geq 0$ with equality somewhere, and satisfies

$$
\left\{\begin{array}{c}
\Delta z-\beta\left(x_{1}, y, c^{\prime}\right) \partial_{1} z+f\left(x_{1}+\tau, u_{\tau}\right)-f\left(x_{1}, u^{\prime}\right) \\
=\left(\beta\left(x_{1}+\tau, y, c\right)-\beta\left(x_{1}, y, c^{\prime}\right)\right) \partial_{1} u_{\tau} \text { in } \Sigma \\
\partial_{\nu} z=0 \text { on } \partial \Sigma
\end{array}\right.
$$

Since $\tau \geq 0, \beta$ is non increasing in $x_{1}, c<c^{\prime}$ and $\partial_{1} u_{\tau} \geq 0$ (from theorem 3), it comes that the second term of the above equation is $\leq 0$. Moreover, $f\left(x_{1}+\tau, u_{\tau}\right) \geq f\left(x_{1}, u_{\tau}\right)$ ( $f$ is non decreasing in $\left.x_{1}\right)$. Hence, there exists a function $c$ in $L^{\infty}$ such that

$$
\left\{\begin{array}{c}
\Delta z-\beta\left(x_{1}, y, c^{\prime}\right) \partial_{1} z+c(x) z \leq 0 \text { in } \Sigma \\
\partial_{\nu} z=0 \text { on } \partial \Sigma
\end{array}\right.
$$

From the strong maximum principle and Hopf lemma, it comes that $z \equiv 0$. That is impossible from the behaviours of $u$ and $u^{\prime}$ near $\pm \infty$.

That achieves the proof of part a) of theorem 4 .

Remark. - From this result, it follows that if $(c, u)$ and $\left(c^{\prime}, u^{\prime}\right)$ are solutions of $(P)$ such that $\max u(0, \cdot)=\max u^{\prime}(0, \cdot)$, then $c=c^{\prime}$. In both cases I and II, for the invariant problem by translation $\left(P_{i n v}\right)$, the speed $c$ solution was unique, and the function $u$ was unique up to translation. The result proved in the next section is the analogous for the non-invariant problem $(P)$.

\subsubsection{Results of partial uniqueness: proof of theorem $4 \mathrm{~b}$ )}

In assertion b) of theorem 4, arguing by contradiction, it suffices to prove that if $(c, u)$ and $\left(c, u^{\prime}\right)$ are solutions of $(P)$ such that $\max _{\bar{\omega}} u(0, \cdot)=\max _{\bar{\omega}} u^{\prime}(0, \cdot)=h \in(0,1)$, then $u \equiv u^{\prime}$. From the hypotheses made for theorem 4, we can apply theorems 1 and 2 of the appendix for the asymptotic behaviours of $u$ and $u^{\prime}$ near $\pm \infty$ : we have already seen in $\S 3.3 .1$ that the behaviours of $u$ and $u^{\prime}$ in $-\infty$ are given by

$$
\left\{\begin{aligned}
u\left(x_{1}, y\right) & =e^{\lambda x_{1}} \phi(y)+o\left(e^{\lambda x_{1}}\right) \\
u^{\prime}\left(x_{1}, y\right) & =C e^{\lambda x_{1}} \phi(y)+o\left(e^{\lambda x_{1}}\right)
\end{aligned} \text { as } x_{1} \rightarrow-\infty\right.
$$

where $C>0$. The eigenvalue $\lambda>0$ and the eigenfunction $\phi>0$ in $\bar{\omega}$ are the unique solutions (up to multiplication of $\phi$ by a positive constant) of

$$
\left\{\begin{array}{c}
\Delta \phi+\left(\lambda^{2}-\lambda \beta_{-}(y, c)+f_{-}^{\prime}(0)\right) \phi=0 \text { in } \omega \\
\partial_{\nu} \phi=0 \text { on } \partial \omega
\end{array}\right.
$$


In the same way, since $\mu_{1}\left(-\Delta-f_{+}^{\prime}(1)\right)=-f_{+}^{\prime}(1)>0$, we can write

$$
\left\{\begin{array}{c}
u\left(x_{1}, y\right)=1-e^{\mu x_{1}} \psi(y)+o\left(e^{\mu x_{1}}\right) \\
u^{\prime}\left(x_{1}, y\right)=1-C^{\prime} e^{\mu x_{1}} \psi(y)+o\left(e^{\mu x_{1}}\right)
\end{array} \text { as } x_{1} \rightarrow+\infty\right.
$$

with $C^{\prime}>0, \mu<0$ and $\psi>0$ on $\bar{\omega}$ solutions of

$$
\left\{\begin{array}{c}
\Delta \psi+\left(\mu^{2}-\mu \beta_{+}(y, c)+f_{+}^{\prime}(1)\right) \psi=0 \text { in } \omega \\
\partial_{\nu} \psi=0 \text { on } \partial \omega
\end{array}\right.
$$

The pair $(\mu, \psi)$ is the unique solution with $\mu<0$ of this problem, up to multiplication by a constant for $\psi$.

Let us recall that $\max _{\bar{\omega}} u(0, \cdot)=\max _{\bar{\omega}} u^{\prime}(0, \cdot)$. We will now translate the function $u$ to the left. For any $s \geq 0$, let us define the function $u_{s}\left(x_{1}, y\right)=u\left(x_{1}+s, y\right)$ in $\bar{\Sigma}$. The function $u_{s}$ clearly satisfies

$$
\begin{gathered}
u_{s}\left(x_{1}, y\right)=c^{\lambda s} e^{\lambda x_{1}} \phi(y)+o\left(c^{\lambda x_{1}}\right) \text { as } x_{1} \rightarrow-\infty \\
u_{s}\left(x_{1}, y\right)=1-e^{\mu s} e^{\mu x_{1}} \psi(y)+o\left(e^{\mu x_{1}}\right) \text { as } x_{1} \rightarrow+\infty
\end{gathered}
$$

Hence, since $u^{\prime}$ is continuous and $0<u^{\prime}<1$ in $\bar{\Sigma}$, it comes that for some $t^{\prime}>0$, we have $u_{t^{\prime}}>u^{\prime}$ in $\bar{\Sigma}$. Let us now decrease $t^{\prime}$ such that, for a real $t \leq t^{\prime}$, one of the following situations occurs:

i) $u_{t} \geq u^{\prime}$ in $\bar{\Sigma}$ with equality somewhere in $\bar{\Sigma}$

ii) $u_{t}>u^{\prime}$ in $\bar{\Sigma}$ and $e^{\mu t}=C^{\prime}$

iii) $u_{t}>u^{\prime}$ in $\bar{\Sigma}$ and $e^{\lambda t}=C$

With the normalization condition on $u$ and $u^{\prime}$ on $\{0\} \times \bar{\omega}$, one of the previous situations must occur for a real $t \geq 0$.

If case i) occurs, then let us note $z=u_{t}-u^{\prime}$. The function $z \geq 0$ satisfies

$$
\left\{\begin{array}{c}
\Delta z-\beta\left(x_{1}, y, c\right) \partial_{1} z+\left(f\left(x_{1}, u_{t}\right)-f\left(x_{1}, u^{\prime}\right)\right) \\
=\left(\beta\left(x_{1}+t, y, c\right)-\beta\left(x_{1}, y, c\right)\right) \partial_{1} u_{t} \\
+\left(f\left(x_{1}, u_{t}\right)-f\left(x_{1}+t, u_{t}\right)\right) \text { in } \Sigma \\
\partial_{\nu} z=0 \text { on } \partial \Sigma
\end{array}\right.
$$

Since $f$ is lipschitz-continuous in $u$ uniformly in $x_{1}$, there exists a function $c$ in $L^{\infty}(\Sigma)$ such that $f\left(x_{1}, u_{t}\right)-f\left(x_{1}, u^{\prime}\right)=c(x) z$. On the other hand, from the monotonicity properties of $\beta$ and $f$ and theorem 3, we have $\left(\beta\left(x_{1}+t, y, c\right)-\beta\left(x_{1}, y, c\right)\right) \partial_{1} u_{t} \leq 0$ and $f\left(x_{1}, u_{t}\right)-f\left(x_{1}+t, u_{t}\right) \leq 0$. Hence the function $z$ satisfies

$$
\left\{\begin{array}{c}
\Delta z-\beta\left(x_{1}, y, c\right) \partial_{1} z+c(x) z \leq 0 \text { in } \Sigma \\
\partial_{\nu} z=0 \text { on } \partial \Sigma
\end{array}\right.
$$

Vol. $14, n^{\circ} 5-1997$ 
As $z \geq 0$ and $z=0$ somewhere in $\bar{\Sigma}$, we conclude from the strong maximum principle and Hopf lemma that $z \equiv 0$ in $\bar{\Sigma}$. Since $\partial_{1} u, \partial_{1} u^{\prime}>0$ in $\bar{\Sigma}$, and from the normalization condition on $\{0\} \times \bar{\omega}$, it comes that necessarly $t=0$ and thus $u \equiv u^{\prime}$.

In case $i i$ ), since $\beta$ is independant of $x_{1}$ near $+\infty$, for $N$ large enough, the function $z=u_{t}-u^{\prime}$ satisfies,

$$
\Delta z-\beta_{+}(y, c) \partial_{1} z+f_{+}^{\prime}(1) z=A+B \text { in }[N,+\infty[\times \omega
$$

where we define $A=f_{+}\left(u_{t}\right)-f\left(x_{1}+t, u_{l}\right)+f\left(x_{1}, u^{\prime}\right)-f_{+}\left(u^{\prime}\right)$ and $B=\left(f_{+}^{\prime}(1)\left(u_{t}-1\right)-f_{+}\left(u_{t}\right)\right)+\left(f_{+}\left(u^{\prime}\right)-f_{+}^{\prime}(1)\left(u^{\prime}-1\right)\right)$.

In case ii), we have implicitely supposed that $z>0$ in $\bar{\Sigma}$ (otherwise, case i) occurs). From the definition of $z$, we can write that $z \leq C^{\prime \prime} e^{\mu x_{1}}$ near $+\infty$. On the other side, from the hypotheses on $f$ near $+\infty$, the term $A$ satisfies

$$
\forall \alpha>0 \quad|A|=O\left(e^{-\alpha x_{1}}\right) \text { as } x_{1} \rightarrow+\infty
$$

Furthermore, from the assumptions on $f_{+}$as $u \rightarrow 1\left(f_{+}\right.$is of class $C^{1, \delta}$ near 1), it follows that

$$
B \leq M\left(1-u_{t}+1-u^{\prime}\right)^{\delta} z \leq M^{\prime} e^{\delta \mu x_{1}} z \text { near }+\infty
$$

Since $\mu_{1}\left(-\Delta_{y}-f_{+}^{\prime}(1)\right)>0$, we can argue as in the proof of theorem 2 of the appendix and conclude that there exist $\rho \geq 0$ and $\epsilon>0$ such that

$$
z=\rho e^{\mu x_{1}} \psi(y)+O\left(e^{(\mu-\epsilon) x_{1}}\right) \text { as } x_{1} \rightarrow+\infty
$$

We recall that the eigenvalue $\mu<0$ and the eigenfunction $\psi>0$ satisfy the eigenvalue problem

$$
\left\{\begin{array}{c}
\Delta \psi+\left(\mu^{2}-\mu \beta_{+}(y, c)+f_{+}^{\prime}(1)\right) \psi=0 \text { in } \omega \\
\partial_{\nu} \psi=0 \text { on } \partial \omega
\end{array}\right.
$$

From the construction of $\mu<0$ in [7], we can add that for $\epsilon^{\prime}>0$ small enough, there exists some pair $\left(\mu_{\epsilon^{\prime}}, \psi_{\epsilon^{\prime}}\right)$ solution of

$$
\left\{\begin{array}{c}
\Delta \psi_{\epsilon^{\prime}}+\left(\mu_{\epsilon^{\prime}}^{2}-\mu_{\epsilon^{\prime}} \beta_{+}(y, c)+f_{+}^{\prime}(1)-\epsilon^{\prime}\right) \psi_{\epsilon^{\prime}}=0 \text { in } \omega \\
\partial_{\nu} \psi_{\epsilon^{\prime}}=0 \text { on } \partial \omega
\end{array}\right.
$$

with $\mu_{\epsilon^{\prime}}<\mu<0$ and $\psi_{\epsilon^{\prime}}>0$ on $\bar{\omega}$. By an argument of compactness and uniqueness, we can say that $\mu_{\epsilon^{\prime}} \rightarrow \mu$ as $\epsilon^{\prime} \rightarrow 0$. Hence, there exists $\epsilon^{\prime}>0$ small enough such that

$$
\mu \quad c<\mu_{\epsilon^{\prime}}<\mu<0 \text { and } \mu(\delta \mid 1)<\mu_{\epsilon^{\prime}}<\mu
$$

Let us now call $w=e^{\mu_{c^{\prime}} x_{1}} \psi_{\epsilon^{\prime}}(y)$. This function $w$ satisfies

$$
\left\{\begin{array}{c}
\Delta w-\beta_{+}(y, c) \partial_{1} w+\left(f_{+}^{\prime}(1)-\epsilon^{\prime}\right) w=0 \text { in } \Sigma \\
\partial_{\nu} w=0 \text { on } \partial \Sigma
\end{array}\right.
$$


Let us now note $v=z-w$. We want to prove that $v \geq 0$ near $+\infty$. This function $v$ satisfies

$$
\Delta v-\beta_{+}(y, c) \partial_{1} v+f_{+}^{\prime}(1) v=A+B-\epsilon^{\prime} e^{\mu_{\epsilon^{\prime} x_{1}}} \psi_{\epsilon^{\prime}}(y) \text { in } \Sigma
$$

From the study above, near $+\infty$, we have $A \leq e^{-\alpha x_{1}}$ for all $\alpha>0$ and $B \leq M^{\prime} e^{\mu(1+\delta) x_{1}}$. As $\mu(1+\delta)<\mu_{\mathrm{e}^{\prime}}<0$ from the choice of $\epsilon^{\prime}$, it follows that for $N^{\prime} \geq N$ large enough,

$$
A+B-\epsilon^{\prime} e^{\mu_{\epsilon^{\prime}} x_{1}} \psi_{\epsilon^{\prime}}(y)<0 \text { in }\left[N^{\prime},+\infty[\times \omega\right.
$$

Since $z>0$ on $\left\{N^{\prime}\right\} \times \bar{\omega}$, we can multiply $w$ by a positive constant such that $v=z-w \geq 0$ on $\left\{N^{\prime}\right\} \times \bar{\omega}$. From the maximum principle and Hopf lemma, and since $f_{+}^{\prime}(1)<0$, we conclude that $v \geq 0$, that is to say

$$
z \geq C^{\prime \prime} e^{\mu_{\epsilon^{\prime}} x_{1}} \psi_{\epsilon^{\prime}}(y) \text { in }\left[N^{\prime},+\infty\left[\times \bar{\omega} \text { with } C^{\prime \prime}>0\right.\right.
$$

On the other side, $\mu-\epsilon<\mu_{\epsilon^{\prime}}<0$. From the behaviour of $z$ as $x_{1} \rightarrow+\infty$ (3.4), that implies that $\rho>0$, but that contradicts the hypothesis made for this case ii). In conclusion, this case can not occur.

If case iii) occurs, then we now come back to the starting point and translate this time $u$ to the right and then to the left such that one of the threee situations analogous to cases i), ii) or iii) occur, but this time for $u_{t^{\prime}}$ and $u^{\prime}$ where $t^{\prime} \leq 0$. We conclude as above that $u \equiv u^{\prime}$ in case i), and that case ii) is impossible. If case iii) occurs then we have $e^{\lambda t}=C$ and $e^{\lambda t^{\prime}}=C$ with $t^{\prime} \leq 0 \leq t$. This clearly implies that $t=t^{\prime}=0$. As $u_{t}>u^{\prime}$ in $\bar{\Sigma}$, that leads to a contradiction with the normalization condition on $\{0\} \times \bar{\omega}$. Thus, case iii) can not occur. Hence, only case i) is possible, that is to say $u \equiv u^{\prime}$. That achieves the proof of theorem $4 \mathrm{~b}$ ).

Remark. - We can not a priori show that if $u$ and $u^{\prime}$ are solutions of $(P)$ with the same speed $c$, then $u \equiv u^{\prime}$ up to translation. Indeed, in order to apply the strong maximum principle to conclude such a conclusion, we can not translate the functions in any way (to the left and to the right) because of the monotonicity properties of $\beta$ and $f$.

\subsubsection{Proof of the last two conditions of theorem 1 a)}

We first consider the two cases I and II ("ignition temperature" or "bistable" cases), and the functions $u_{ \pm}$solutions of

$\left(P_{ \pm}\right) \quad\left\{\begin{array}{c}\Delta u_{ \pm}-\beta_{ \pm}\left(y, c_{ \pm}\right) \partial_{1} u_{ \pm}+f_{ \pm}\left(u_{ \pm}\right)=0 \text { in } \Sigma \\ \partial_{\nu} u_{ \pm}=0 \text { on } \partial \Sigma \\ u_{ \pm}(-\infty, \cdot)=0, u_{ \pm}(+\infty, \cdot)=1\end{array}\right.$ 
We proved in $\S 2.1 .1$ that $c_{-} \leq c_{+}$. It only remains to show that $c_{-}<c_{+}$ if $f_{-} \not \equiv f_{+}$and $\left|f_{-}(1-s)-f_{+}(1-s)\right|=O\left(s^{1+\delta}\right)$ as $s \rightarrow 0$ for some $\delta>0$. Let us suppose that $c_{-}=c_{+}$, which we call $c$, and show that $f_{-} \equiv f_{+}$. Like in $\S 2.1 .1$, the hypothesis $\beta_{-}(\cdot, c) \not \equiv \beta_{+}(\cdot, c)$ would yield a contradiction. Hence, we have $\beta_{-}(\cdot, c) \equiv \beta_{+}(\cdot, c)$, which we rename $\beta(\cdot, c)$. The functions $u_{ \pm}$satisfy the following equations:

$$
\Delta u_{ \pm}-\beta(y, c) \partial_{1} u_{ \pm}+f_{ \pm}\left(u_{ \pm}\right)=0 \text { in } \Sigma
$$

In each case $I$ and II, the behaviours of $u_{ \pm}$as $x_{1} \rightarrow \pm \infty$ are given by

$$
\begin{gathered}
u_{ \pm}\left(x_{1}, y\right)=C_{ \pm} e^{\lambda_{ \pm} x_{1}} \phi_{ \pm}(y)+o\left(e^{\lambda_{ \pm} x_{1}}\right) \text { as } x_{1} \rightarrow-\infty \\
u_{ \pm}\left(x_{1}, y\right)=1-C_{ \pm}^{\prime} e^{\mu_{ \pm} x_{1}} \psi_{ \pm}(y)+o\left(e^{\mu_{ \pm} x_{1}}\right) \text { as } x_{1} \rightarrow+\infty
\end{gathered}
$$

where $\lambda_{ \pm}>0, \mu_{ \pm}<0, \phi_{ \pm}, \psi_{ \pm}>0$ on $\bar{\omega}$ are solutions of

$$
\begin{gathered}
\left\{\begin{array}{c}
\Delta \phi_{ \pm}+\left(\lambda_{ \pm}^{2}-\lambda_{ \pm} \beta(y, c)+f_{ \pm}^{\prime}(0)\right) \phi_{ \pm}=0 \text { in } \omega \\
\partial_{\nu} \phi_{ \pm}=0 \text { on } \partial \omega
\end{array}\right. \\
\left\{\begin{array}{c}
\Delta \psi_{ \pm}+\left(\mu_{ \pm}^{2}-\begin{array}{c}
\left.\mu_{ \pm} \beta(y, c)+f_{ \pm}^{\prime}(1)\right) \psi_{ \pm}=0 \text { in } \omega \\
\partial_{\nu} \psi_{ \pm}=0 \text { on } \partial \omega
\end{array}\right.
\end{array}\right.
\end{gathered}
$$

Like in $\S 2.1 .1$, we have $\lambda_{-}>\lambda_{+}>0$ and $\mu_{+} \leq \mu_{-}<0$ with equality if and only if $f_{-}^{\prime}(0)=f_{+}^{\prime}(0)$ and $f_{-}^{\prime}(1)=f_{+}^{\prime}(1)$ respectively.

We can now argue exactly as above in 33.3 .2 . Up to translation, we can suppose that $\max _{\bar{\omega}} u_{+}(0, \cdot)=\max _{\bar{\omega}} u_{-}(0, \cdot)$. Translating the function $u_{+}$ to the left enough to be greater than $u_{-}$in $\Sigma$, and then to the right, we would necessarly be led to consider the three cases i), ii), and iii) of $\$ 3.3 .2$. Case i) where $u_{+} \geq u_{-}$with equality somewhere is treated as in $\S 2.1 .1$ by the maximum principle and Hopf lemma. To conclude in case ii) (which occurs if and only if $f_{-}^{\prime}(1)=f_{+}^{\prime}(1)$, i.e. $\left.\mu:=\mu_{-}=\mu_{+}\right)$, we introduce the function $z=u_{+}-u_{-}$, and write

$$
\begin{aligned}
\Delta z- & \beta(y, c) \partial_{1} z+f_{+}^{\prime}(1) z \\
= & \left(f_{-}\left(u_{-}\right)-f_{+}\left(u_{-}\right)\right) \\
& +\left(\left(f_{+}\left(u_{-}\right)-f_{+}^{\prime}(1) u_{-}\right)-\left(f_{+}\left(u_{+}\right)-f_{+}^{\prime}(1) u_{+}\right)\right.
\end{aligned}
$$

From the assumptions on $f_{+}(s)$ and $f_{-}(s)$ as $s \rightarrow 1$, the second member is $O\left(e^{\mu(1+\delta) x_{1}}\right)$ as $x_{1} \rightarrow+\infty$. That allows to apply the arguments of the end of the proof of theorem $4 \mathrm{~b}$ ).

Case iii) is treated in the same way as in $§ 3.3 .2$. 
In short, we conclude that $u_{-} \equiv u_{+}$up to translation. Since $u_{-}$takes its values on $(0,1)$, it follows that $f_{-} \equiv f_{+}$.

In case I ("ignition temperature"), when $f_{+}^{\prime}(1)<f_{-}^{\prime}(1)<0$, then we have $c_{-}<c_{+}$. Indeed, since $f_{-}(s) \equiv f_{+}(s) \equiv 0$ in a neighbourhood of $s=0$, we can argue as above by a change of variables $x_{1}^{\prime}=-x_{1}$ (let us remark that the equality of the asymptotic behaviours as $x_{1}^{\prime} \rightarrow-\infty$, i.e. case iii, can not occur because we assumed that $f_{+}^{\prime}(1)<f_{-}^{\prime}(1)<0$ ).

\section{ACKNOWLEDGEMENTS}

The author would like to thank Professor Berestycki for his helpful support and suggestions in this work.

\section{APPENDIX: EXPONENTIAL BEHAVIOURS}

\section{Main results}

In this appendix, one studies the properties of any solution $u$ of the following elliptic semilinear problem

$$
\left\{\begin{array}{c}
\Delta u-\beta\left(x_{1}, y, c\right) \partial_{1} u+f\left(x_{1}, y, u\right)=0 \text { in } 2 \\
\partial_{\nu} u=0 \text { on } \partial \Sigma \\
u(-\infty, \cdot)=0, u>0
\end{array}\right.
$$

We first mention the works [1], [6], [7], [15] and [18] on the solutions of similar equations, but with no dependance on $x_{1}$. Problem $\left(P^{\prime}\right)$ is more general that problem $(P)$ since the non-linearity $f$ may depend this time on the transversal variable $y$. The same assumptions of regularity as in the introduction are made on $f$ and $\beta$. Moreover, $f$ is lipschitz-continuous with respect to all variables, and $f\left(x_{1}, y, 0\right)=f\left(x_{1}, y, 1\right)=0$ for all $\left(x_{1}, y\right) \in \bar{\Sigma}$. One systematically assumes that $f$ is non decreasing in $x_{1}$ and $\beta$ is non increasing in $x_{1}$.

Moreover, one introduces additional assumptions on the behaviours of $\beta$ and $f$ as $x_{1} \rightarrow \pm \infty$. Some of these hypotheses correspond to those of theorems 3 and 4 given in the general introduction.

Firstly, $\forall \alpha>0$,

$$
\left\{\begin{array}{l}
\left|f\left(x_{1}, y, u\right)-f_{-}(y, u)\right|=O\left(e^{\alpha x_{1}}\right) \text { as } x_{1} \rightarrow-\infty \\
\left|f\left(x_{1}, y, u\right)-f_{+}(y, u)\right|=O\left(e^{-\alpha x_{1}}\right) \text { as } x_{1} \rightarrow+\infty
\end{array}\right.
$$

uniformly in $(y, u) \in \bar{\omega} \times[0,1]$. 
Secondly, the functions $\partial_{u} f_{-}(y, 0)$ and $\partial_{u} f_{+}(y, 1)$ exist. Let us note $d(y, s)=f_{+}(y, 1-s)+\partial_{u} f_{+}(y, 1) s$. We assume that there exist constants $M, \delta$ and $s_{0}>0$ such that

$$
\left\{\begin{array}{c}
\left|f_{-}(y, s)-\partial_{u} f_{-}(y, 0) s\right| \leq M s^{1+\delta} \quad \forall y \in \bar{\omega} \quad \forall 0 \leq s \leq s_{0} \\
\left|f_{+}(y, 1-s)+\partial_{u} f_{+}(y, 1) s\right| \leq M s^{1+\delta} \quad \forall y \in \bar{\omega} \quad \forall 0 \leq s \leq s_{0} \\
\left|d(y, s)-d\left(y, s^{\prime}\right)\right| \leq M\left|s-s^{\prime}\right|\left(s+s^{\prime}\right)^{\delta} \quad \forall y \in \bar{\omega} \quad \forall 0 \leq s, s^{\prime} \leq s_{0}
\end{array}\right.
$$

Thirdly, $\beta$ decays faster than some exponential near $\pm \infty$. More precisely, there exist reals $\delta_{ \pm}>0$ such that

$$
\left\{\begin{array}{c}
\left|\beta\left(x_{1}, y, c\right)-\beta_{+}(y, c)\right|=O\left(e^{-\delta_{+} x_{1}}\right) \text { as } x_{1} \rightarrow+\infty \\
\left|\beta\left(x_{1}, y, c\right)-\beta_{-}(y, c)\right|=O\left(e^{\delta_{-} x_{1}}\right) \text { as } x_{1} \rightarrow-\infty
\end{array}\right.
$$

uniformly in $(y, c) \in \bar{\omega} \times \mathbb{R}$.

In order to study the exponential behaviour of $u$ near $-\infty$, let us note $\mu_{1}$ the principal eigenvalue of the elliptic limit operator $-\Delta_{y}-\partial_{u} f_{-}(y, 0)$ with Neumann boundary conditions and $\sigma$ the principal eigenfunction, i.e. $\sigma>0$ on $\bar{\omega}$ and satisfies

$$
\left\{\begin{array}{c}
-\Delta_{y} \sigma-\partial_{u} f_{-}(y, 0) \sigma=\mu_{1} \sigma \text { in } \omega \\
\partial_{\nu} \sigma=0 \text { on } \partial \omega
\end{array}\right.
$$

THEOREM 1. - Assume that $\beta$ is non increasing in $x_{1}, f$ is non decreasing in $x_{1}$ and the hypotheses above are satisfied. In each of the following cases,

a) $\mu_{1}>0$

b) $\mu_{1}<0$

c) $\mu_{1}=0, f\left(x_{1}, y, s\right)-\partial_{u} f_{-}(y, 0) s \leq 0 \forall 0 \leq s \leq s_{0} \forall\left(x_{1}, y\right) \in \bar{\Sigma}$ and $\int \beta_{-}(y, c) \sigma(y)^{2}>0$,

if $u$ is a positive solution of $\left(P^{\prime}\right)$ then

i) $\left\{\begin{array}{c}u\left(x_{1}, y\right)=e^{\lambda x_{1}} \phi(y)+o\left(e^{\lambda x_{\perp}}\right) \\ \nabla u\left(x_{1}, y\right)=\nabla\left(e^{\lambda x_{1}} \phi(y)\right)+o\left(e^{\lambda x_{1}}\right)\end{array}\right.$ as $x_{1} \rightarrow-\infty$

or $i i)\left\{\begin{array}{c}u\left(x_{1}, y\right)=e^{\lambda x_{1}}\left(-x_{1} \phi(y)+\phi_{0}(y)\right)+o\left(e^{\lambda x_{1}}\right) \\ \nabla u\left(x_{1}, y\right)=\nabla\left(e^{\lambda x_{1}}\left(-x_{1} \phi(y)+\phi_{0}(y)\right)+o\left(e^{\lambda x_{1}}\right)\right.\end{array}\right.$ as $x_{1} \rightarrow-\infty$ with $\lambda>0, \phi>0$ on $\bar{\omega}$ solution of

$$
\left\{\begin{array}{c}
\Delta_{y} \phi+\left(\lambda^{2}-\lambda \beta_{-}(y, c)+\partial_{u} f_{-}(y, 0)\right) \phi=0 \text { in } \omega \\
\partial_{\nu} \phi=0 \text { on } \partial \omega
\end{array}\right.
$$

The real $\lambda>0$ is unique in cases a) and c). Besides, case ii) may only occur if $\mu_{1}<0$ (but this is not sufficient, see [7]). 
For the study of the functions near $+\infty$, let us note $\left(P^{\prime \prime}\right)$ the following problem

$$
\left\{\begin{array}{c}
\Delta u-\beta\left(x_{1}, y, c\right) \partial_{1} u+f\left(x_{1}, y, u\right)=0 \text { in } \Sigma \\
\partial_{\nu} u=0 \text { on } \partial \Sigma \\
u(+\infty, \cdot)=1, u<1
\end{array}\right.
$$

Let us now note $\mu_{1}^{\prime}$ the principal eigenvalue of the elliptic operator $-\Delta_{y}-\partial_{u} f_{+}(y, 1)$ with Neumann boundary conditions and $\sigma^{\prime}$ its principal eigenfunction. The behaviour near $+\infty$ is given by the similar theorem

THEOREM 2. - Assume that $\beta$ is non increasing in $x_{1}, f$ non decreasing in $x_{1}$ and the hypotheses above are satisfied. In each of the following cases

a) $\mu_{1}^{\prime}>0$

b) $\mu_{1}^{\prime}<0$

c) $\mu_{1}^{\prime}=0, f\left(x_{1}, y, s\right)-\partial_{u} f_{+}(y, 1)(s-1) \geq 0 \forall 1-s_{0} \leq s \leq 1$, $\forall\left(x_{1}, y\right) \in \bar{\Sigma}$ and $\int \beta_{+}(y, c) \sigma^{\prime}(y)^{2}<0$,

if $u$ is a solution $<1$ of $\left(P^{\prime \prime}\right)$ then

i) $\left\{\begin{aligned} u\left(x_{1}, y\right) & =1-e^{\mu x_{1}} \psi(y)+o\left(e^{\mu x_{1}}\right) \\ \nabla u\left(x_{1}, y\right) & =-\nabla\left(e^{\mu x_{1}} \psi(y)\right)+o\left(e^{\mu x_{1}}\right)\end{aligned}\right.$ as $x_{1} \rightarrow+\infty$

or $i i)\left\{\begin{aligned} u\left(x_{1}, y\right) & =1-e^{\mu x_{1}}\left(x_{1} \psi(y)+\psi_{0}(y)\right)+o\left(e^{\mu x_{1}}\right) \\ \nabla u\left(x_{1}, y\right) & =-\nabla\left(e^{\mu x_{1}}\left(x_{1} \psi(y)+\psi_{0}(y)\right)\right)+o\left(e^{\mu x_{1}}\right)\end{aligned}\right.$ as $x_{1} \rightarrow+\infty$

with $\mu<0, \psi>0$ on $\bar{\omega}$ solution of

$$
\left\{\begin{array}{c}
\Delta_{y} \psi+\left(\mu^{2}-\mu \beta_{+}(y, c)+\partial_{u} f_{+}(y, 1)\right) \psi=0 \text { in } \omega \\
\partial_{\nu} \psi=0 \text { on } \partial \omega
\end{array}\right.
$$

The real $\mu<0$ is unique in cases a) and c). Besides, case ii) may only occur if $\mu_{1}^{\prime}<0$.

\section{Proofs of theorems 1 and 2}

For the proof of theorem 1 , let us note $\Sigma_{-}=\mathbb{R}_{-}^{*} \times \omega$. Let us consider a positive function $u$ of problem $\left(P^{\prime}\right)$. In other words, $u$ is a positive solution in $W_{l o c}^{2, p}\left(\Sigma^{-}\right)$of the elliptic problem

$$
\left\{\begin{array}{c}
L u=0 \text { in } \Sigma_{-} \\
\partial_{\nu} u=0 \text { on } \mathbb{R}_{-}^{*} \times \partial \omega \\
u(-\infty, \cdot)=0, u>0
\end{array}\right.
$$

where $L$ is the elliptic operator $L=\Delta+\beta\left(x_{1}, y, c\right) \partial_{1}+c(x)$ and the functions $x \mapsto \beta\left(x_{1}, y, c\right)$ and $x \mapsto c(x)=\frac{f\left(x_{1}, y, u\right)}{u}$ are in $L^{\infty}\left(\Sigma_{-}\right)$ 
from the assumptions on $\beta$ and $f$. From theorem 3.2 of [7], which is a consequence of the Harnack inequalities up to the boundary for positive functions, it follows that there exist constants $a>0, c_{1}>0$ and $c_{2}>0$ only depending on $\sup _{x \in \Sigma^{-}}\left|\beta\left(x_{1}, y, c\right)\right|$, sup $\operatorname{su}_{x \in \Sigma^{-}}|c(x)|$ and $\omega$ such that

$$
C_{1} e^{a x_{1}} \leq u\left(x_{1}, y\right) \leq C_{2} e^{-a x_{1}} \text { in } \bar{\Sigma}
$$

Hence, $u$ can not decay faster than any exponential. Let us now show that $u$ actually decays faster than some exponentially decreasing function in $\Sigma_{-}$. At first, we need the following intermediate lemma

LEMma 1. - If there exists a function $a_{0}$ defined in $\bar{\omega}$ which satisfies one of the following assertions:

Case 1: $f\left(x_{1}, y, s\right) \leq a_{0}(y) s$ for $0 \leq s \leq s_{0}\left(s_{0}>0\right)$ and $-x_{1}$ large enough, and the first eigenvalue $\mu_{1}\left(-\Delta-a_{0}\right)$ is $>0$.

Case 1': $f\left(x_{1}, y, s\right) \leq a_{0}(y)$ s for $0 \leq s \leq s_{0}\left(s_{0}>0\right)$ and for $-x_{1}$ large enough, the first eigenvalue $\mu_{1}\left(-\Delta-a_{0}\right)=0$ and $\int \beta_{-}(y, c) \sigma^{2}(y)>0$ where $\sigma$ is the principal eigenfunction of $-\Delta-a_{0}$.

Case 2: $f\left(x_{1}, y, s\right) \geq a_{0}(y) s$ for $0 \leq s \leq s_{0}\left(s_{0}>0\right)$ and for $-x_{1}$ large enough, and the first eigenvalue $\mu_{1}\left(-\Delta-a_{0}\right)$ is $<0$,

then there exist $\epsilon>0, C_{1}>0$ such that

$$
u(x)+|\nabla u(x)| \leq C_{1} e^{\epsilon x_{1}} \text { in } \Gamma
$$

Proof of lemma 1 in cases 1 and 1':

From the results of [7], there exist an eigenvalue $\lambda_{-}>0$ and an eigenfunction $\phi_{-}>0$ on $\bar{\omega}$ solutions of

$$
\left\{\begin{array}{c}
\left(-\Delta-a_{0}(y)-\lambda_{-}^{2}+\lambda_{-} \beta_{-}(y, c)\right) \phi_{-}=0 \text { in } \omega \\
\partial_{\nu} \phi_{-}=0 \text { on } \partial \omega
\end{array}\right.
$$

We can solve the same eigenvalue problem with $\beta_{x_{1}}(y, c):=\beta\left(x_{1}, y, c\right)$ for any $x_{1}$ in case 1 and for $-x_{1}$ large enough in case $1^{\prime}$ in such a way that $\int \beta\left(x_{1}, y, c\right) \sigma(y)^{2}>0$ (this is allowed since $\int \beta\left(x_{1}, y, c\right) \sigma(y)^{2} \rightarrow$ $\int \beta_{-}(y, c) \sigma(y)^{2}$ as $\left.x_{1} \rightarrow-\infty\right)$. There exist thus eigenvalues $\lambda_{x_{1}}>0$ and eigenfunctions $\phi_{x_{1}}>0$ on $\bar{\omega}$ such that

$$
\left\{\begin{array}{c}
\left(-\Delta-a_{0}(y)-\lambda_{x_{1}}^{2}+\lambda_{x_{1}} \beta_{x_{1}}(y, c)\right) \phi_{x_{1}}=0 \text { in } \omega \\
\partial_{\nu} \phi_{x_{1}}=0 \text { on } \partial \omega
\end{array}\right.
$$

If we suppose that the functions $\phi_{x_{1}}$ are normalized in $L^{2}(\omega)$ $\left(\left\|\phi_{x_{1}}\right\|_{L^{2}}=1\right)$ and $\phi_{x_{1}}>0$, then the pairs $\left(\lambda_{x_{1}}, \phi_{x_{1}}\right)$ are unique. By 
an argument of compactness and uniqueness, and using the results of [7], we can even add that $\lambda_{x_{1}} \rightarrow \lambda_{-}$and $\phi_{x_{1}} \rightarrow \phi_{-}$in $W^{2,2}(\omega)$ as $x_{1} \rightarrow-\infty$.

Let us call $L_{0}$ the elliptic operator $L_{0}=\Delta-\beta\left(x_{1}, y, c\right) \partial_{1}+a_{0}(y)$. In cases 1 and $1^{\prime}$, since $u \rightarrow 0$ as $x_{1} \rightarrow-\infty$, we have for $x_{1} \leq-A$ ( $A$ large enough),

$$
\left.\left.L_{0}(u) \geq \Delta u-\beta\left(x_{1}, y, c\right) \partial_{1} u+f\left(x_{1}, y, u\right)=0 \text { in }\right]-\infty,-A\right] \times \omega
$$

Let us note $\lambda=\lambda_{-A}, \phi=\phi_{-A}$ and $v=e^{\lambda x_{1}} \phi(y)$. Since $\beta$ is non increasing in $x_{1}$ and from the equation satisfied by $\lambda$ and $\phi$, we have

$$
\left.\left.L_{0}(v)=\left(\beta(-A, y, c)-\beta\left(x_{1}, y, c\right)\right) \partial_{1} v \leq 0 \text { in }\right]-\infty,-A\right] \times \omega
$$

and moreover $w=u-C e^{\lambda x_{1}} \phi(y) \leq 0$ on $\{-A\} \times \bar{\omega}$ if $C>0$ is large enough. We have thus $L_{0}(w) \geq 0$ and we want to prove that $w \leq 0$ in ]$-\infty,-A] \times \omega$. But the zero-order term $a_{0}(y)$ is not negative. Let us recall that the first eigenvalue $\sigma>0$ on $\bar{\omega}$ is such that

$$
\left\{\begin{array}{c}
\left(-\Delta-a_{0}(y)\right) \sigma=\mu_{1} \sigma \text { in } \omega \\
\partial_{\nu} \sigma=0 \text { on } \partial \omega
\end{array}\right.
$$

Let us define a function $z$ such that $w=\sigma z$, we can then write

$$
\left\{\begin{array}{c}
\left.\left.0 \leq \frac{L_{0} w}{\sigma}=\Delta z+\frac{2}{\sigma} \nabla \sigma \cdot \nabla_{y} z-\beta\left(x_{1}, y, c\right) \partial_{1} z-\mu_{1} z \text { in }\right]-\infty,-A\right] \times \omega \\
\left.\left.\partial_{\nu} z=0 \text { on }\right]-\infty,-A\right] \times \partial \omega
\end{array}\right.
$$

Since $-\mu_{1} \leq 0$ and $z \leq 0$ at $-\infty$ and on $\{-A\} \times \bar{\omega}$, the maximum principle and Hopf lemma imply that $z \leq 0$ in $]-\infty,-A] \times \bar{\omega}$. Hence,

$$
\left.\left.u \leq C e^{\lambda x_{1}} \phi(y) \text { in }\right]-\infty,-A\right] \times \bar{\omega}
$$

Since $u$ satisfies an elliptic equation and $\partial_{\nu} u=0$, the classical a priori estimates up to the boundary yield

$$
\left.\left.\left|\nabla u\left(x_{1}, y\right)\right| \leq C^{\prime} \max _{\substack{\left|\xi-x_{1}\right| \leq 1 / 2 \\ \eta \in \bar{\omega}}} u(\xi, \eta) \text { in }\right]-\infty,-A-1\right] \times \bar{\omega}
$$

The proof of formula (2) is thus ended if we just change $C$ and $C^{\prime}$.

Proof of lemma $I$ in case 2. - This time, for $A$ large, the function $u$ satisfies

$$
\left\{\begin{array}{c}
\left.\left.L_{0}(u)=\Delta u-\beta\left(x_{1}, y, c\right) \partial_{1} u+a_{0}(y) u \leq 0 \text { in }\right]-\infty,-A\right] \times \omega \\
\left.\left.\partial_{\nu} u=0 \text { on }\right]-\infty,-A\right] \times \partial \omega
\end{array}\right.
$$

Vol. 14, $\mathbf{n}^{\circ}$ 5-1997. 
Since the functions $\beta$ and $a_{0}$ are bounded and since $\mu_{1}<0$, we can apply lemma 3.1 of [7] and write that there exist constants $\epsilon>0$ and $C>0$ such that

$$
\int_{-\infty}^{-N} \int_{\omega} u \leq C e^{-\epsilon N} \quad \forall N \geq A, N \text { integer }
$$

Next, from the classical a priori estimates up to the boundary, we have:

$$
u\left(x_{1}, y\right)+\left|\nabla u\left(x_{1}, y\right)\right| \leq C_{2} \int_{x_{1}-1}^{x_{1}+1} \int_{\omega} u
$$

This achieves the proof of lemma 1 in case 2 .

Let us recall that we have introduced $\mu_{1}$ the first eigenvalue of the elliptic operator $-\Delta-\partial_{u} f_{-}(y, 0)$ and $\sigma$ the principal eigenfunction of this operator. We can now precise the exponential decay of the solutions $u$ of $\left(P^{\prime}\right)$ from the following lemma :

Lemma 2. - There exist constants $C_{1}, C, a, b>0$ such that

$$
\begin{gathered}
C_{1} e^{\alpha x_{1}} \leq u\left(x_{1}, y\right) \leq C e^{b x_{1}} \text { in } \Sigma_{-} \\
\left|\nabla u\left(x_{1}, y\right)\right| \leq C_{1} e^{b x_{1}} \text { in } \Sigma_{-}
\end{gathered}
$$

in each of the following cases

- case l: $\mu_{1}>0$

- case $1^{\prime}: \mu_{1}=0, h\left(x_{1}, y, s\right)=f\left(x_{1}, y, s\right)-\partial_{u} f_{-}(y, 0) s \leq 0 \quad \forall 0 \leq s \leq s_{0}$, $\forall\left(x_{1}, y\right)$ and $\int_{\omega} \beta_{-}(y, c) \sigma^{2}(y)>0$

- case 2: $\mu_{1}<0$

Proof. - The left part of inequality (3) corresponds to the left part of (1). In order to prove the right part of $(3)$ and $\left(3^{\prime}\right)$, we will come down to lemma 1.

In case $1^{\prime}$, we apply directly case $1^{\prime}$ of lemma 1 .

In case 1 , let $\epsilon>0$ such that $\mu_{1}\left(-\Delta-\partial_{u} f_{-}(y, 0)-\epsilon\right)>0$ and define $a_{0}(y)=\partial_{u} f_{-}(y, 0)+\epsilon$. It only remains to prove that $f\left(x_{1}, y, u\right) \leq a_{0}(y) u$ in the neighbourhood of $-\infty$. We can write,

$f\left(x_{1}, y, u\right)-a_{0}(y) u=\left(f\left(x_{1}, y, u\right)-f_{-}(y, u)\right)+\left(f_{-}(y, u)-\partial_{u} f_{-}(y, 0) u-\epsilon u\right)$

From now on, $A$ denotes a positive constant large enough. Let us recall that $u \geq C_{1} e^{a x_{1}}$ (from (1)) and $\left|f\left(x_{1}, y, u\right)-f_{-}(y, u)\right| \leq e^{(a+1) x_{1}}$ if $x_{1} \leq-A$, $\forall(y, u)$ (by the hypothesis made on $f$, with $\alpha-a+1)$. Hence,

$$
\left|f\left(x_{1}, y, u\right)-f_{-}(y, u)\right| \leq \epsilon / 2 u \text { if } x_{1} \leq-A
$$


On the other hand, since $u \rightarrow 0$ as $x_{1} \rightarrow-\infty$, we have

$$
\left|f_{-}(y, u)-\partial_{u} f_{-}(y, 0) u\right| \leq M u^{1+\delta} \leq \epsilon / 2 u \text { if } x_{1} \leq-A
$$

Hence,

$$
f\left(x_{1}, y, u\right)-a_{0}(y) u \leq 0 \text { if } x_{1} \leq-A
$$

Case 2 can be treated in the same way.

End of the proof of theorem 1

Here, we will make use of several results on the asymptotic behaviour of solutions of elliptic equations in cylinders, in the linear and non linear cases. These results were proved in more general situations in [1] and [15], and were recalled in [7] and [6].

At first, from theorem 4.2 of [7] and from [1], if $u^{0}$ is a solution of the linearized problem

$$
\left(P_{-}^{l}\right) \quad\left\{\begin{array}{c}
\Delta u^{0}-\beta_{-}(y, c) \partial_{1} u^{0}+\partial_{u} f_{-}(y, 0) u^{0}=0 \text { in } \Sigma_{-} \\
\partial_{\nu} u^{0}=0 \text { on } \mathbb{R}_{-}^{*} \times \partial \omega
\end{array}\right.
$$

and if $u^{0}$ satisfies $\left|u^{0}\left(x_{1}, y\right)\right| \leq C_{2} e^{b x_{1}}$ in $\Sigma_{-}$for some $C_{2}, b>0$, then there exist an exponential solution $w=e^{\lambda x_{1}} \psi\left(x_{1}, y\right)$ of the linearized problem $\left(P_{-}^{l}\right)$ and $\epsilon>0$ such that

$$
\left\{\begin{array}{c}
u^{0}\left(x_{1}, y\right)=e^{\lambda x_{1}} \psi\left(x_{1}, y\right)+O\left(e^{(\lambda+\epsilon) x_{1}}\right) \\
\nabla u^{0}\left(x_{1}, y\right)=\nabla\left(e^{\lambda x_{1}} \psi\left(x_{1}, y\right)\right)+O\left(e^{(\lambda+\epsilon) x_{1}}\right)
\end{array} \text { as } x_{1} \rightarrow-\infty\right.
$$

where $\lambda \geq b$ and $\psi\left(x_{1}, y\right)=\left(-x_{1}\right)^{k} \psi_{k}(y)+\cdots+\psi_{0}(y) \not \equiv 0$.

Next, let us remark that $u$ actually satisfies the unhomogeneous problem $\left(P_{-}^{\prime}\right)$ :

$$
\left\{\begin{array}{c}
\Delta u-\beta_{-}(y, c) \partial_{1} u+\partial_{u} f_{-}(y, 0) u \\
=r(x):=\left(\beta\left(x_{1}, y, c\right)-\beta_{-}(y, c)\right) \partial_{1} u \\
-\left(f\left(x_{1}, y, u\right)-f_{-}(y, u)\right) \\
+\left(\partial_{u} f_{-}(y, 0) u-f_{-}(y, u)\right) \text { in } \Sigma_{-} \\
\partial_{\nu} u=0 \text { on } \mathbb{R}_{-}^{*} \times \partial \omega
\end{array}\right.
$$

From theorem 4.3 of [7], if there is constants $\alpha, C>0$ such that

$$
|r(x)| \leq C e^{\alpha x_{1}} \text { in } \Sigma_{-}
$$

then $u=u^{0}+u^{*}$ where $u^{0}$ is a solution of $\left(P_{-}^{l}\right)$ and $u^{*}$ solution of $\left(P_{-}^{\prime}\right)$. Besides, for all $\epsilon>0$, there exists a constant $C_{\epsilon}>0$ such that

$$
\left|u^{*}\left(x_{1}, y\right)\right|+\left|\nabla u^{*}\left(x_{1}, y\right)\right| \leq C_{\epsilon} e^{(\alpha-\epsilon) x_{1}} \text { in } \Sigma_{-}
$$


We will now argue as in [7]. Let

$$
\tau_{0}=\sup \left\{\tau: \exists C_{\tau} \text { such that } u\left(x_{1}, y\right) \leq C_{\tau} e^{\tau x_{1}} \text { in } \Sigma \text { - }\right\}
$$

From the inequalities (3), we have $0<b \leq \tau_{0} \leq a$. For any $\tau<\tau_{0}$, we have $0 \leq u\left(x_{1}, y\right) \leq C_{\tau} e^{\tau x_{1}}$ and even $|\nabla u| \leq C_{\tau}^{\prime} e^{\tau x_{1}}$ from the classical elliptic estimates. From the hypotheses on the behaviours of $\beta$ and $f$ as $x_{1} \rightarrow-\infty$, and $f_{-}$as $u \rightarrow 0$, it follows that

$$
\begin{aligned}
|r(x)| & \leq C_{-} e^{\delta-x_{1}} C_{\tau}^{\prime} e^{\tau x_{1}}+C e^{(\tau \mid 1) x_{1}}+M C_{\tau}^{1 \mid \delta} e^{\tau(1 \mid \delta) x_{1}} \\
& \leq C_{\tau}^{\prime \prime} e^{m(\tau) x_{1}} \text { as } x_{1} \rightarrow-\infty
\end{aligned}
$$

where $m(\tau)=\min \left(\tau+\delta_{-}, \tau+1, \tau(1+\delta)\right)>\tau$.

Hence, from the results above, we have for some $D_{\tau}>0$,

$$
\left|u^{*}\left(x_{1}, y\right)\right|+\left|\nabla u^{*}\left(x_{1}, y\right)\right| \leq D_{\tau} e^{\frac{\tau+m(\tau)}{2} x_{1}} \text { in } \Sigma_{-}
$$

When $\tau \nearrow \tau_{0}$, we have $\frac{\tau+m(\tau)}{2} \rightarrow \frac{\tau_{0}+m\left(\tau_{0}\right)}{2}>\tau_{0}$. Hence, there exist constants $\beta>0$ and $C>0$ such that

$$
\left|u^{*}\left(x_{1}, y\right)\right|+\left|\nabla u^{*}\left(x_{1}, y\right)\right| \leq C e^{\left(\tau_{0}+3\right) x_{1}} \text { in } \Sigma_{-}
$$

As a consequence, for any $\tau<\tau_{0}$,

$$
\left|u^{0}\left(x_{1}, y\right)\right| \leq C_{\tau} e^{\tau x_{1}}+C e^{\left(\tau_{0}+\beta\right) x_{1}} \leq\left(C_{\tau}+C\right) e^{\tau x_{1}} \text { in } \Sigma_{-}
$$

From the first result recalled above, we can write

$$
\left\{\begin{array}{l}
u^{0}\left(x_{1}, y\right)=e^{\lambda x_{1}} \psi\left(x_{1}, y\right)+o\left(e^{\lambda x_{1}}\right) \\
\nabla u^{0}=\nabla\left(e^{\lambda x_{1}} \psi\left(x_{1}, y\right)\right)+o\left(e^{\lambda x_{1}}\right)
\end{array} \text { as } x_{1} \rightarrow-\infty\right.
$$

with $\lambda>0$ and $w=e^{\lambda x_{1}} \psi\left(x_{1}, y\right)$ solution of the linearized problem $\left(P_{-}^{l}\right)$. The definition of $\tau_{0}$ and the remarks above imply that $\lambda=\tau_{0}$. Hence,

$$
\left\{\begin{array}{c}
u\left(x_{1}, y\right)=e^{\lambda x_{1}} \psi\left(x_{1}, y\right)+o\left(e^{\lambda x_{1}}\right) \\
\nabla u\left(x_{1}, y\right)=\nabla\left(e^{\lambda x_{1}} \psi\left(x_{1}, y\right)\right)+o\left(e^{\lambda x_{1}}\right)
\end{array} \text { as } x_{1} \rightarrow-\infty\right.
$$

Let us note that we can even change $o\left(e^{\lambda x_{1}}\right)$ in the behaviours of $u^{0}$ and thus of $u$ by $O\left(c^{(\lambda+\epsilon) x_{1}}\right)$ for some $c>0$. Since $u>0$, we kave $\psi_{k}>0$ and from theorem 2.4 of [7] on the exponential solutions of linear elliptic problems, it comes that

i) $\psi\left(x_{1}, y\right)=\phi(y)$

or ii) $\psi\left(x_{1}, y\right)=-x_{1} \phi(y)+\phi_{0}(y)$ 
with a function $\phi>0$ on $\bar{\omega}$ solution of

$$
\left\{\begin{array}{c}
\Delta \phi+\left(\lambda^{2}-\lambda \beta_{-}(y, c)+\partial_{u} f_{-}(y, 0)\right) \phi=0 \text { in } \omega \\
\partial_{\nu} \phi=0 \text { on } \partial \omega
\end{array}\right.
$$

Moreover, case ii) can only occur if $\mu_{1}<0$, but this is not sufficient, and this corresponds to the only case where the pair $(\lambda>0, \phi>0)$ solution of the previous problem is not unique (see [7]).

This achieves the proof of theorem 1 .

Obviously, the proof of theorem 2 is similar. It only suffices to make the change of variables $x_{1} \rightarrow-x_{1}$ and $u \rightarrow 1-u$ to come down to a study of a behaviour near $-\infty$.

\section{REFERENCES}

[1] S. AGMon and L. Nirenberg, Properties of solutions of ordinary differential equations in Banach space, Comm. Pure Appl. Math., Vol. 16, 1963, pp. 121-239.

[2] D. G. ARonson and H. F. WeInBERger, Multidimensional nonlinear diffusions arising in population genetics, Adv. in Math., Vol. 30, 1978, pp. 33-58.

[3] H. BERESTYCKI and B. LARROUTUROU, Quelques aspects mathématiques de la propagation des flammes prémélangées, Nonlinear p.d.e. and their applications, Collège de France seminar, Vol. 10, Brézis and Lions eds, Pitman Longman, Harbow, UK, 1990.

[4] H. BERESTYCKI and B. LARRouturou, A semilinear elliptic equation in a strip arising in a two-dimensional flame propagation model, J. Reine Angew. Math., Vol. 396, 1989, pp. $14-40$.

[5] H. Berestycki, B. Larrouturou and P. L. Lions, Multidimensional traveling-wave solutions of a flame propagation model, Arch. Rat. Mech. Anal., Vol. 111, 1990, pp. 33-49.

[6] H. Berestycki, B. Larrouturou and J. M. Roquejoffre, Stability of traveling fronts in a curved flame model, Part I, Linear Analysis, Arch. Rat. Mech. Anal., Vol. 117, 1992, pp. $97-117$.

[7] H. Berestyckı and L. Nirenberg, Travelling fronts in cylinders, Ann. Inst. H. Poincaré, Analyse Non Linéaire, Vol. 9, 5, 1992, pp. 497-572.

[8] H. BERESTyCKI and L. Nirenberg, On the method of moving planes and the sliding method, Bol. da Soc. Braseleira de Matematica, Vol. 22, 1991, pp. 1-37.

[9] P. C. FIFE and J. B. MCLEOD, The approach of solutions of non-linear diffusion equations to traveling front solutions, Arch. Rat. Mech. Anal., Vol. 65, 1977, pp. 335-361.

[10] F. HAMEL, Reaction-diffusion problems in cylinders with no invariance by translation, part I: Small perturbations, Ann. Inst. H. Poincaré, Analyse Non Linéaire, Vol. 14, 1997, pp. 457-498.

[11] F. HAMEL, Formules min-max pour les vitesses d'ondes progressives multidimensionnelles. preprint Labo. Ana. Num., n ${ }^{\circ}$ 96032, Univ. Paris VI, France, 1996.

[12] Ja. I. KANEL', Stabilization of solution of the Cauchy problem for equations encountred in combustion theory, Mat. Sbornik., Vol. 59, 1962, pp. 245-288.

[13] A. N. Kolmogorov, I. G. Petrovsky and N. S. Piskunov, Etude de l'équation de la diffusion avec croissance de la quantité de matière et son application à un problème biologique, Bulletin Université d'Etat à Moscow (Bjul. Moskowskogo Gos. Univ.), Série internationale, section A.1, 1937, pp. 1-26.

[14] A. Liñan, Fluid Dynanic Aspects of Combustion Theory, course of IAC. Mauro Picone. Rome, 1989. 
[15] A. PazY, Asymptotic expansions of solutions of ordinary differential equations in Hilbert space, Arch. Rat. Mech. Anal., Vol. 24, 1967, pp. 193-218.

[16] G. I. Sivashinsky, Instabilities, pattern formation and turbulence in flames, Ann. Rev. Fluid Mech., Vol. 15, 1983, pp. 179-199.

[17] J. M. VEGA, On the uniqueness of multidimensional travelling fronts of some semilinear equations, J. Math. Anal. Appl., Vol. 177, 1993, pp. 481-490.

[18] J. M. VEGA, The asymptotic behavior of the solutions of some semilinear elliptic equations in cylindrical domains, J. Diff. Eq., Vol. 102, 1993, pp. 119-152.

[19] V. A. VOLPERT and A. I. VOLPERT, Existence and Stability of Stationary Solutions for a Class of Semilinear Parabolic Systems, Comm. in Part. Diff. Eq., Vol. 18, 1993, pp. 2051-2069.

[20] F. Williams, Combustion Theory, Addison-Wesley, reading MA, 1983.

[21] X. XIN, Existence and Uniqueness of Travelling Waves in a Reaction-Diffusion Equation with Combustion Nonlinearity, Idiana Univ. Math. J., Vol. 40, No 3, 1991.

[22] X. XIN, Existence of planar flame fronts in convective diffusive periodic media, Arch. Rat. Mech. Analysis, Vol. 121, 1992, pp. 205-233.

[23] X. XIN, Existence and stability of travelling waves in periodic media governed by a bistable nonlinearity, J. Dyn. Diff. Fq., Vol. 3, 1991, pp. 541-573.

[24] X. XIN, Existence and non existence of travelling waves and reaction-diffusion front propagation in periodic media, J. Statist. Phys., Vol. 73, 1993, pp. 893-926.

$[25]$ J. B. Zeldovich and D. A. FranK-KAMENETSKII, A theory of thermal propagation of flame, Acta physiochimica URSS, Vol. 9, 1938. English translation in Dynamics of curved fronts, R. Pelcé ed., Perspectives in Physics Series, Academic Press, New York, 1988, pp. 131-140.

(Manuscript received June 7, 1995.) 